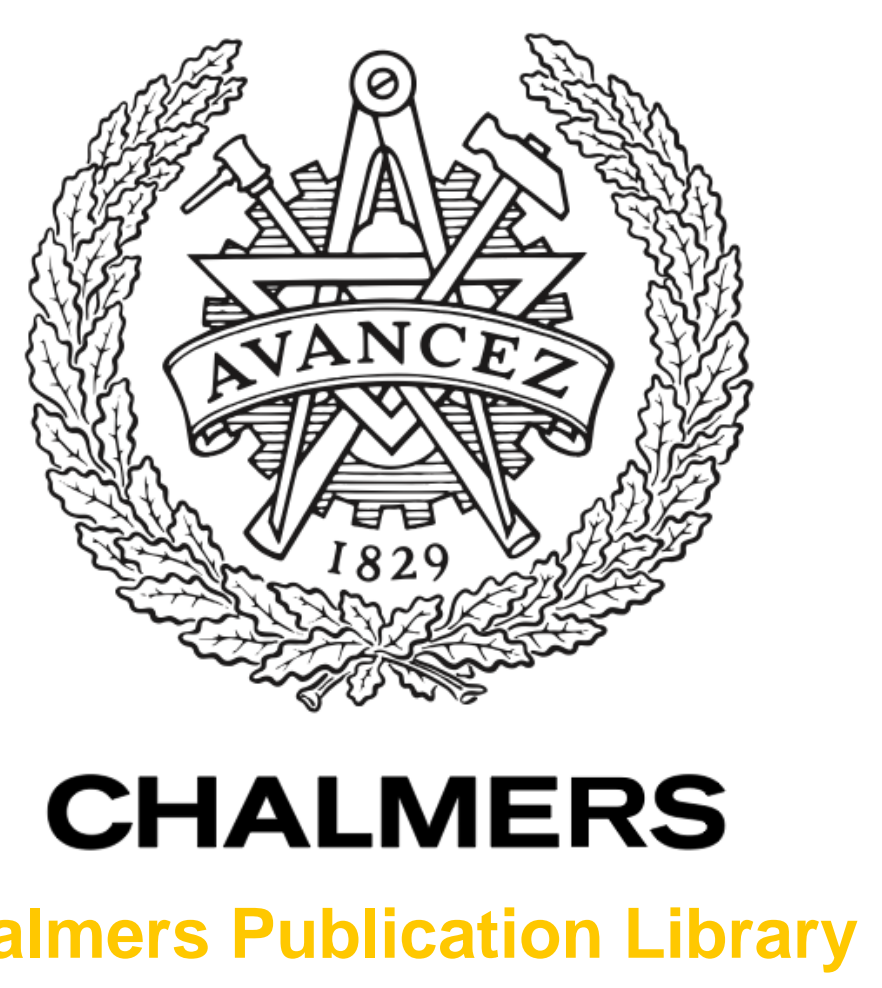

Chalmers Publication Library

\title{
Semi-analytical analysis of the isolation to moving-load induced ground vibrations by trenches on a poroelastic half-space
}

This document has been downloaded from Chalmers Publication Library (CPL). It is the author's version of a work that was accepted for publication in:

Journal of Sound and Vibration (ISSN: 0022-460X)

Citation for the published paper:

Cao, Z. ; Cai, Y. ; Boström, A. et al. (2012) "Semi-analytical analysis of the isolation to moving-load induced ground vibrations by trenches on a poroelastic half-space". Journal of Sound and Vibration, vol. 331(4), pp. 947-961.

http://dx.doi.org/10.1016/j.jsv.2011.09.009

Downloaded from: http://publications.lib.chalmers.se/publication/156985

Notice: Changes introduced as a result of publishing processes such as copy-editing and formatting may not be reflected in this document. For a definitive version of this work, please refer to the published source. Please note that access to the published version might require a subscription.

Chalmers Publication Library (CPL) offers the possibility of retrieving research publications produced at Chalmers University of Technology. It covers all types of publications: articles, dissertations, licentiate theses, masters theses, conference papers, reports etc. Since 2006 it is the official tool for Chalmers official publication statistics. To ensure that Chalmers research results are disseminated as widely as possible, an Open Access Policy has been adopted.

The CPL service is administrated and maintained by Chalmers Library. 


\title{
Semi-analytical analysis of the isolation to moving-load induced ground vibrations by trenches on a poroelastic half-space
}

\author{
Zhigang Cao ${ }^{a, b, *}$, Yuanqiang Cai ${ }^{a}$, Anders Boström ${ }^{b}$, Jianguo Zheng ${ }^{c}$ \\ ${ }^{a}$ MOE Key Laboratory of Soft Soils and Geoenvironmental Engineering, Zhejiang University, Hangzhou 310027, \\ PR China \\ ${ }^{b}$ CHARMEC, Department of Applied mechanics, Chalmers University of Technology, SE-41296 Gothenburg, Sweden \\ ${ }^{c}$ CHINA JK INSTITUTE OF ENGINEERING INVESTIGATION AND DESIGN, Xi'an, 710004, PR China
}

\begin{abstract}
A semi-analytical model is proposed to investigate the screening efficiency of trenches to moving-load induced ground vibrations. The ground is modeled as a fully saturated poroelastic half-space governed by Biot's dynamic poroelastic theory. The trenches are obtained by placing three rectangular elastic layers with appropriate width upon the poroelastic half-space. By Helmholtz decomposition, the displacement fields of the elastic layers are decomposed into three scalar potentials. Analytical solutions are obtained based on Fourier transform and Fourier series in the transformed domain. The time-domain results are obtained by the fast Fourier transform (FFT). The different performances of trenches on a saturated poroelastic half-space and a single-phase elastic half-space to the moving load-induced ground vibration are identified. It is found that the discrepancy of the screening efficiencies between the two models becomes significant when the load speed approaches the Rayleigh wave speed of the ground surface. Also, some parametric studies for the screening efficiency of the trench on the poroelastic half-space are presented.
\end{abstract}

\section{Introduction}

Vibrations induced by railway traffic, machine operations, or pile driving are a major concern for civil engineers as it can cause annoyance to residents or even damage to adjacent structures. Wave 
barriers are needed in some cases to prevent or reduce the adverse effects of ground vibrations to the concerned structure.

Many investigations concerning vibration isolations have been conducted by different kinds of methods. To name a few, Segol et al. [1] used the finite element method (FEM) and a two-dimensional slab-type model for studying vibration screening by open trenches and in-filled trenches in layered soils. Haupt [2] employed FEM in investigating the use of solid obstacles of different shapes for passive, as well as active, isolation. Takemiya [3] and With et al. [4] investigated the isolation efficiency of countermeasures along a railway line to the train-induced ground vibrations by FEM as well as field tests. Yang and Hung [4] presented a parametric study of wave barriers for the train-induced ground vibrations and the effects of parameters such as the dimensions of the open and filled trenches and modulus of the in-filled material on the screening efficiency were investigated. Hung et al. [6] studied the effectiveness of different vibration countermeasures, including open trenches, in-filled trenches, and wave impeding blocks to the train-induced ground vibration with a 2.5D finite/infinite element approach. Using the boundary element method (BEM), Emad and Manolis [7], Beskos et al. [8], Dasgupta et al. [9], Ahmad and Al-Hussaini [10] and Klein et al. [11] investigated the isolation efficiency of countermeasures to incident waves or harmonic point loads. Some experimental work has also been conducted, such as Woods [12], who performed an experimental investigation on the effectiveness of open trenches in reducing the amplitude of vertical ground vibrations, a scale experiment in water by Liao and Sangrey [13], and an open trench experiment by Klein et al. [11]. Analytical treatments of the vibration isolation problems have also been provided. Aviles and Sanchez-Sesma [14] and Boroomand and Kaynia [15, 16] developed analytical models to study the amplitude reduction 
behind a row of circular solid piles in an elastic half-space when subjected to incident waves. Based on their studies, Cai et al. [17] and Xu et al. [18] studied a row of rigid piles as countermeasures in a saturated poroelastic soil medium for reducing the ground vibration to incident waves or a moving point load, and it was found that the isolation performance of a pile barrier in a poroelastic soil was better than that in an elastic soil medium. Karlström and Boström [19] proposed an analytical approach to investigate the screening effectiveness of open trenches on a layered elastic half-space to the train-induced ground vibrations at both sub- and super-critical train speeds.

In the work mentioned above, the ground is often simplified as a single-phase elastic medium, and the effects of underground water are neglected. Under some circumstances, the coupling effects between the soil skeleton and underground water can affect the wave propagation significantly in the saturated soil medium. Existing studies (Cai et al. [17], Xu et al. [18]) show that the screening effect of piles in a saturated poroelastic soil medium is quite different from those in a single-phase elastic soil medium in some cases. Thus, the saturated poroelastic soil model can be essential in the investigation of ground vibration reduction by countermeasures. On the other hand, an open trench is known as an efficient and economic countermeasure to reduce the high-frequency ground vibration components and is widely used in the engineering field. The isolation performance of open trenches on a saturated poroelastic half-space to moving-load induced ground vibrations has not been studied yet. Thus, it is of significance to investigate the screening effectiveness of trenches more exactly by a saturated poroelastic soil model, including the coupling effect between the soil skeleton and the water.

In this paper, an analytical model is proposed to investigate the screening efficiency of open trenches to the ground vibration generated by a moving rectangular load. The ground is modeled as 
a fully saturated poroelastic half-space, taking into account the coupling between the soil skeleton and the fluid. Analytical solutions are obtained based on Fourier transforms and Fourier series. The main contribution of this paper is to identify the different performances of trenches on a poroelastic half-space and an elastic half-space to moving loads with various load speeds. Furthermore, the effects of the coupling between the soil skeleton and the fluid on the screening efficiency of the trench are further investigated. Finally, the effects of the distance between the trench and the moving-load on the screening efficiency are analyzed.

\section{Governing equations and solutions}

The geometrical details of the trench model are shown in Fig. 1. The ground is modeled as a fully saturated poroelastic half-space. The trenches are obtained by placing three rectangular elastic layers with appropriate widths on the poroelastic half-space. The widths of the outer layers are chosen large enough so as to approximate a half-infinite layer. The moving load is represented by a moving rectangular load. The trenches are placed symmetrically at both sides of the moving load. For simplicity the bottom of the trenches are assumed to coincide with the top of the poroelastic half-space. The present procedure can probably be generalized to the case with poroelastic top layers so that the trenches are situated in poroelastic half-space. Generalizations to a layered half-space are straightforward, see Karlström and Boström [20].

\subsection{Governing equations and solutions for the elastic layers}

The Lamé equations for the elastic layers are given as:

$$
\left(\lambda_{j}+2 \mu_{j} \nabla \nabla \mathbf{u}_{j}\right) \mu_{j} \nabla \rtimes\left(\nabla \times \times_{j}\right) \ddot{\rho} \mathbf{u}_{j}
$$

where $\lambda_{j}, \mu_{j}$ are the Lamé constants and $\rho_{j}$ is the density of the elastic layers. The gradient 
operator is defined as $\nabla=\left\{\partial_{x}, \partial_{y}, \partial_{z}\right\}$. The displacement vector $\mathbf{u}_{j}$ is defined as $\mathbf{u}_{j}=\left\{u_{j}, v_{j}, w_{j}\right\}$ for the $x, y$ and $z$ direction, where $j=\mathrm{e}$ represents the middle embankment layer and $j=b^{+}, b^{-}$ denotes the banks outside. The traction on a plane with the normal direction $\mathbf{e}_{k}(k=x, y, z)$ can be obtained by

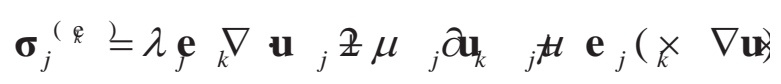

The interface between the elastic layers and the poroelastic half-space is assumed to be perfectly bonded, and then the displacement and traction vectors are continuous. At $z=0$ this gives:

$$
\begin{gathered}
\mathbf{u}=\left\{\begin{array}{cc}
\mathbf{u}_{\mathrm{e}} & |y|<a_{\mathrm{e}}, \\
\mathbf{u}_{\mathrm{b}^{ \pm}} & b_{1}<|y|<b_{2},
\end{array}\right. \\
\boldsymbol{\sigma}^{\left(\mathrm{e}_{\mathrm{e}}\right)}=\left\{\begin{array}{cc}
\boldsymbol{\sigma}_{\mathrm{e}}^{\left(\mathrm{e}_{2}\right)} & |y|<a_{\mathrm{e}}, \\
\boldsymbol{\sigma}_{\mathrm{b}^{ \pm}}^{\left(\mathrm{e}_{2}\right)} & b_{1}<|y|<b_{2}, \\
0 & a_{\mathrm{e}}<|y|<b_{1} \text { and }|y|>b_{2},
\end{array}\right.
\end{gathered}
$$

where $\mathbf{u}, \boldsymbol{\sigma}^{\left(\mathbf{e}_{z}\right)}$ are the displacement and traction vector of the saturated poroelastic half-space. The boundary conditions on the sides of the embankment layer $\left(|y|=a_{\mathrm{e}}\right)$ are designed in a special way in order to enable the series expansion of the displacement fields according to Karlström and Boström [18]:

$$
v_{\mathrm{e}}=0, \partial_{y} u_{\mathrm{e}}=0 \partial_{y} w_{\mathrm{e}}=0-d \underset{\mathrm{e}}{\mathrm{Z}}<
$$

This boundary condition is obtained by choosing $v_{\mathrm{e}}$ to be zero, which is artificial, and combining the shear stresses condition on the sides of the embankment layers. This is equivalent to:

$$
v_{\mathrm{e}}=0, \sigma_{x y \mathrm{e}}=0 \sigma \bar{z}_{z}=\quad 0-d \mathrm{y}_{\mathrm{y}} \mathrm{K}
$$

It was stated in Karlström and Boström [19] that the artificial boundary condition $v_{\mathrm{e}}=0$ gives a good approximation to the natural boundary condition $\sigma_{y \mathrm{e}}=0$ when the wavelength is much larger than the thickness of the elastic layer, and only vertical loads are applied. That the depth of 
the trenches are less than the wavelength may seem to indicate that they have a small effect on the reduction of vibrations, but as our numerical examples will show the trenches have noticeable effects.

The boundary conditions on the top of the embankment $\left(z=-d_{\mathrm{e}}\right)$ can be expressed as

$$
\begin{aligned}
& \sigma_{z z e}=\left\{\begin{array}{cc}
Q & |x-V t| \leq a,|y| \leq b, \\
0 & \text { else where }
\end{array}\right. \\
& \sigma_{y z e}=0 \\
& \sigma_{x z \mathrm{e}}=0
\end{aligned}
$$

where $Q$ is the amplitude of the load pressure, $V$ is the load speed, $a$ and $b$ are half the load width and length, respectively. For the banks $\left(\mathrm{b}^{+}, \mathrm{b}^{-}\right)$, the traction on the top of the elastic layers vanishes:

$$
\boldsymbol{\sigma}_{\mathrm{b}^{ \pm}}^{\mathbf{e}_{2}}=0, \quad b_{1} \leq|y| \leq b_{2} .
$$

Similar boundary conditions are also used at the sides of the banks:

$$
v_{\mathrm{b}^{ \pm}}=0, \partial_{y} u_{\mathrm{b}^{ \pm}}=0, \partial_{y} w_{\mathrm{b}^{ \pm}}=0 \quad-d_{\mathrm{b}}<z<0
$$

By decomposing the displacement fields of the embankment $(j=\mathrm{e})$ and banks $\left(j=\mathrm{b}^{ \pm}\right)$in three scalar potentials, analytical solutions to the equations of motion (1) can be obtained by

$$
\mathbf{u}_{j}=\nabla \varphi_{j}+\nabla \times\left(\mathbf{e}_{z} \psi_{\mathrm{SHj}}\right)+\nabla \times \nabla \times\left(\mathbf{e}_{z} \psi_{\mathrm{SVj}}\right)
$$

where $\varphi_{j}, \psi_{\mathrm{SH} j}$ and $\psi_{\mathrm{Svj}}$ are potentials for longitudinal, horizontal transverse and vertical transverse waves, respectively, which satisfy scalar wave equations.

Fourier transforms are applied with respect to $t$ and $x$. The Fourier transform pair for the time $t$ is defined as

$$
\begin{aligned}
& \left.\tilde{f}(\omega)=\int_{-\infty}^{+\infty} f(t)\right)^{\omega t} \mathrm{e} \\
& f(t)=\frac{1}{2 \pi} \int_{-\infty}^{+\infty} \tilde{f}(\omega) \mathrm{e}^{-\mathrm{i} \omega t} \mathrm{~d} \omega .
\end{aligned}
$$


The Fourier transform pair for the space coordinate $x$ is defined as

$$
\begin{aligned}
& \bar{g}(q)=\int_{-\infty}^{+\infty} g(x) \mathrm{e}^{-\mathrm{i} q x} \mathrm{~d} x, \\
& g(x)=\frac{1}{2 \pi} \int_{-\infty}^{+\infty} \bar{g} \phi \text { jqx } q \prime
\end{aligned}
$$

The doubled transformed fields (with respect to the time $t$ and the space coordinate $x$ ) are denoted by a hat.

As the boundaries on the vertical sides of the embankment layers and the banks are designed in a special way, the displacement fields in the finite domain can be developed by adopting Fourier series expansions. According to Karlström and Boström [19], the scalar potentials in the layers $j=\mathrm{e}$ and $b^{ \pm}$can be given as follows.

$$
\begin{aligned}
& \hat{\varphi}_{j}=\sum_{m=0}^{\infty}\left(D_{1 m}^{j} \sin \left(h_{j m}^{\mathrm{p}} z\right)+E_{1 m}^{j} \cos \left(h_{j m}^{\mathrm{p}} z\right)\right) \cos \left(p_{j m} T_{j}(y)\right), \\
& \hat{\psi}_{\mathrm{SH} j}=\sum_{m=1}^{\infty}\left(D_{2 m}^{j} \sin \left(h_{j m}^{\mathrm{s}} z\right)+E_{2 m}^{j} \cos \left(h_{j m}^{\mathrm{s}} z\right)\right) \sin \left(p_{j m} T_{j}(y)\right), \\
& \hat{\psi}_{\mathrm{SV} j}=\sum_{m=0}^{\infty}\left(E_{3 m}^{j} \sin \left(h_{j m}^{\mathrm{s}} z\right)-D_{3 m}^{j} \cos \left(h_{j m}^{\mathrm{s}} z\right)\right) \cos \left(p_{j m} T_{j}(y)\right) .
\end{aligned}
$$

Here the coefficients $D_{n m}^{j}$ and $E_{n m}^{j}$ are unknown amplitudes in the embankment layer $j=e$ and in the banks $j=b^{ \pm}$, where $n=1,2$ and 3 give the amplitudes for the P, SH and SV waves, respectively. Furthermore, $c_{j}^{\mathrm{P}}=\left(\left(\lambda_{j}+2 \mu_{j}\right) / \rho_{j}\right)^{1 / 2}$ is the pressure wave velocity and $c_{j}^{\mathrm{S}}=\left(\mu_{j} / \rho_{j}\right)^{1 / 2}$ is the shear wave velocity. The wave numbers are $k_{j}^{\mathrm{P}}=\omega / c_{j}^{\mathrm{P}}$ and $k_{j}^{\mathrm{S}}=\omega / c_{j}^{\mathrm{S}}$. Corresponding wave numbers in the $z$ direction are $h_{j m}^{\mathrm{P}}=\left(k_{j}^{\mathrm{P} 2}-q^{2}-p_{j m}^{2}\right)^{1 / 2}$ and $h_{j m}^{\mathrm{S}}=\left(k_{j}^{\mathrm{S} 2}-q^{2}-p_{j m}^{2}\right)^{1 / 2}$, where the roots are defined so that $\operatorname{Im} h_{j m}^{\mathrm{P}} \geq 0$ and $\operatorname{Im} h_{j m}^{\mathrm{S}} \geq 0$. The trigonometric arguments $T_{j}(y)$ and $p_{j m}$ depend on the region and are given in Table 1.

The constants $D_{n m}^{j}$ and $E_{n m}^{j}$ are to be determined for each domain through the boundary 
conditions. Due to the symmetry of the displacement field $\mathbf{u}_{\mathrm{e}}$ about $y=0$, the constants are equal in the left and right banks and only one of the banks needs to be considered (the right bank in this case). Making use of Eq. (12), the displacements of the embankment and banks can be obtained as

$$
\begin{gathered}
\hat{u}_{j}=\sum_{m=0}^{\infty} \hat{u}_{j}(\mathrm{z}) \operatorname{c~о~} \Phi\left({ }_{j m} T_{j}\right. \\
\hat{v}_{j}=\sum_{m=1}^{\infty} \hat{v}_{j m}(z) \sin \left(p_{j m} T_{j}(y)\right), \\
\hat{w}_{j}=\sum_{m=0}^{\infty} \hat{w}_{j m}(z) \cos \left(p_{j m} T_{j}(y)\right),
\end{gathered}
$$

where

$$
\begin{aligned}
& \hat{u}_{j m}(z)=\sum_{n=1}^{3} \alpha_{n m}^{j}\left(D_{n m}^{j} \sin \left(k_{n m}^{j} z\right)+E_{n m}^{j} \cos \left(k_{n m}^{j} z\right)\right), \\
& \hat{v}_{j m}(z)=\sum_{n=1}^{3} \beta_{n m}^{j}\left(D_{n m}^{j} \sin \left(k_{n m}^{j} z\right)+E_{n m}^{j} \cos \left(k_{n m}^{j} z\right)\right), \\
& \hat{w}_{j m}(z)=\sum_{n=1}^{3} \gamma_{n m}^{j}\left(D_{n m}^{j} \cos \left(k_{n m}^{j} z\right)-E_{n m}^{j} \sin \left(k_{n m}^{j} z\right)\right) .
\end{aligned}
$$

The displacement coefficients $\alpha_{n m}^{j}, \quad \beta_{n m}^{j}, \quad \gamma_{n m}^{j}$ and $k_{n m}^{j}$ can be found in Table 2 .

Substituting Eq. (20)-(22) into Eq. (2), the stresses in the embankment layer and the banks are expressed as:

$$
\begin{aligned}
& \hat{\sigma}_{x z j}=\sum_{m=0}^{\infty} \hat{\sigma}_{x z j m}(z) \cos p_{j m} T_{j}(y), \\
& \hat{\sigma}_{y z j}=\sum_{m=0}^{\infty} \hat{\sigma}_{y z j m}(z) \sin p_{j m} T_{j}(y), \\
& \hat{\sigma}_{z z j}=\sum_{m=0}^{\infty} \hat{\sigma}_{z z j m}(z) \cos p_{j m} T_{j}(y),
\end{aligned}
$$

where 


$$
\begin{aligned}
& \hat{\sigma}_{x z j m}(z)=\sum_{n=1}^{3} \mu_{j} \xi_{n m}^{j}\left(D_{n m}^{j} \cos \left(k_{n m}^{j} z\right)-E_{n m}^{j} \sin \left(k_{n m}^{j} z\right)\right), \\
& \hat{\sigma}_{y z j m}(z)=\sum_{n=1}^{3} \mu_{j} \zeta_{n m}^{j}\left(D_{n m}^{j} \cos \left(k_{n m}^{j} z\right)-E_{n m}^{j} \sin \left(k_{n m}^{j} z\right)\right), \\
& \hat{\sigma}_{z z j m}(z)=\sum_{n=1}^{3} \mu_{j} \eta_{n m}^{j}\left(D_{n m}^{j} \sin \left(k_{n m}^{j} z\right)+E_{n m}^{j} \cos \left(k_{n m}^{j} z\right)\right) .
\end{aligned}
$$

Here some more coefficients are introduced:

$$
\begin{gathered}
\xi_{n m}^{j}=k_{n m}^{j} \alpha_{n m}^{j}+\mathrm{i} q \gamma_{n m}^{j}, \\
\zeta_{n m}^{j}=k_{n m}^{j} \beta_{n m}^{j}-p_{j m} \gamma_{n m}^{j}, \\
\eta_{n m}^{j}=\left(\left(k_{j}^{\mathrm{S}} / k_{j}^{\mathrm{P}}\right)^{2}-2\right)\left(i q \alpha_{n m}^{j}+p_{j m} \beta_{n m}^{j}\right)-\left(k_{j}^{\mathrm{S}} / k_{j}^{\mathrm{P}}\right)^{2} k_{n m}^{j} \gamma_{n m}^{j} .
\end{gathered}
$$

2.2 Coupling between the saturated poroelastic half-space and the elastic layer.

Biot's theory is employed for the saturated poroelastic half-space. Neglecting the apparent mass density, the linearized dynamic equations of motion for a fully saturated poroelastic solid are given by Biot [21] as:

$$
\begin{gathered}
\mu u_{i, j j}+\left(\lambda+\alpha^{2} M+\mu\right) u_{j, j i}+\alpha M w_{j, j i}=\rho \ddot{u}_{i}+\rho_{\mathrm{f}} \ddot{w}_{i}, \\
\alpha M u_{j, j i}+M w_{j, j i}=\rho_{\mathrm{f}} \ddot{u}_{i}+m_{1} \ddot{w}_{i}+\frac{\rho_{\mathrm{f}} g}{k_{\mathrm{d}}} \dot{w}_{i},
\end{gathered}
$$

where $u_{i}, w_{i}(i=x, y, z)$ are the solid displacement components and fluid displacement related to the solid displacement along the $x, y, z$ directions; dots on $u_{i}$ and $w_{i}$ indicate differentials with respect to time $t ; \lambda$ and $\mu$ are Lamé constants of the poroelastic half-space; $\alpha$ and $M$ are Biot's parameters accounting for compressibility of the two-phased material; $\rho=n \rho_{\mathrm{f}}+(1-n) \rho_{\mathrm{s}}$, where $\rho_{\mathrm{f}}$ and $\rho_{\mathrm{s}}$ are the mass densities of the fluid and solid and $n$ is the porosity; $m_{1}=\frac{\rho_{\mathrm{f}}}{n} \cdot k_{\mathrm{d}}$ denotes the coefficient of permeability, and the parameter $b$ is introduced and defined as $b=\frac{\rho_{\mathrm{f}} g}{k_{\mathrm{d}}}$. The constitutive relations can be expressed as: 


$$
\begin{gathered}
\sigma_{i j}=\lambda \delta_{i j} \theta+\mu\left(u_{i, j}+u_{j, i}\right)-\alpha \delta_{i j} p, \\
p_{\mathrm{f}}=-\alpha M \theta+M \varsigma,
\end{gathered}
$$

where

$$
\varsigma=-w_{i, i},
$$

and $\theta=u_{i, i}$ is the solid strain; $\sigma_{i j}$ is the total stress component of the bulk material; $p_{\mathrm{f}}$ is the pore water pressure.

The governing equations for the poroelastic half-space can be solved by triple Fourier transform with respect to $x, y$ and $t$. The Fourier transform pair for the space coordinate $y$ is defined as

$$
\begin{gathered}
\bar{g}(p)=\int_{-\infty}^{+\infty} g(y) \mathrm{e}^{-\mathrm{i} p y} \mathrm{~d} y, \\
g(y)=\frac{1}{2 \pi} \int_{-\infty}^{+\infty} \bar{g}(p) \mathrm{e}^{\mathrm{i} p y} \mathrm{~d} p .
\end{gathered}
$$

The governing equations are solved in the transformed domain. Cai et al. [22] give the specific steps of the solution process. The solutions can be expressed as

$$
\begin{gathered}
\overline{\hat{p}}=A \mathrm{e}^{-\gamma_{1} z}+B \mathrm{e}^{-\gamma_{2} z}, \\
\overline{\hat{u}}_{x}=-\frac{\mathrm{i}}{q}\left\{\left[\chi_{1}+a_{1}\left(q^{2}-L_{1}^{2}\right)\right] A \mathrm{e}^{-\gamma_{1} z}+\left[\chi_{2}+a_{2}\left(q^{2}-L_{2}^{2}\right)\right] B \mathrm{e}^{-\gamma_{2} z}+\left(C \gamma_{3}+D p\right) \mathrm{e}^{-\gamma_{3} z}\right\}, \\
\overline{\hat{u}}_{y}=-\mathrm{i} p\left(a_{1} A \mathrm{e}^{-\gamma_{1} z}+a_{2} B \mathrm{e}^{-\gamma_{2} z}\right)+\mathrm{i} D \mathrm{e}^{-\gamma_{3} z}, \\
\overline{\hat{u}}_{z}=\gamma_{1} a_{1} A \mathrm{e}^{-\gamma_{1} z}+\gamma_{2} a_{2} B \mathrm{e}^{-\gamma_{2} z}+C \mathrm{e}^{-\gamma_{3} z} .
\end{gathered}
$$

The stresses in the poroelastic half-space can be obtained with Eqs. (39)-(42) and (34) as:

$$
\begin{gathered}
\overline{\hat{\sigma}}_{x z}=\frac{\mu \mathrm{i}}{q}\left[\gamma_{1} g_{1} A \mathrm{e}^{-\gamma_{1} z}+\gamma_{2} g_{2} B \mathrm{e}^{-\gamma_{2} z}+\left(C \gamma_{3}^{2}+C q^{2}+D \gamma_{3} p\right) \mathrm{e}^{-\gamma_{3} z}\right], \\
\overline{\hat{\sigma}}_{y z}=\mu \mathrm{i}\left[2 p \gamma_{1} a_{1} A \mathrm{e}^{-\gamma_{1} z}+2 p \gamma_{2} a_{2} B \mathrm{e}^{-\gamma_{2} z}+\left(C p-D \gamma_{3}\right) \mathrm{e}^{-\gamma_{3} z}\right],
\end{gathered}
$$




$$
\overline{\hat{\sigma}}_{z z}=g_{3} A \mathrm{e}^{-\gamma_{1} z}+g_{4} B \mathrm{e}^{-\gamma_{2} z}-2 \mu \gamma_{3} C \mathrm{e}^{-\gamma_{3} z} .
$$

In Eqs. (39)-(45), $A, B, C$ and $D$ are constants to be determined and the other coefficients can be found in the Appendix.

At the interface between the saturated poroelastic half-space and the finite ground layers, the Fourier transform is applied with respect to $y$ to the interface conditions (Eq. (4)), and the following equations are obtained:

$$
\begin{aligned}
& \frac{\mu \mathrm{i}}{q}\left\{\gamma_{1} g_{1} A+\gamma_{2} g_{2} B+\left[C\left(\gamma_{3}^{2}+q^{2}\right)+D \gamma_{3} p\right]\right\}= \\
& \sum_{m=0}^{\infty} \hat{\sigma}_{x z e m}(0) f_{\mathrm{em}}^{\left(-a_{\mathrm{e}}, a_{\mathrm{e}}\right)}(p)+\sum_{m=0}^{\infty} \hat{\sigma}_{x z \mathrm{~b}^{-} m}(0) f_{\mathrm{b}^{-} m}^{\left(-b_{2},-b_{1}\right)}(p)+\sum_{m=0}^{\infty} \hat{\sigma}_{x z \mathrm{~b}^{+} m}(0) f_{\mathrm{b}^{+} m}^{\left(b_{1}, b_{2}\right)}(p), \\
& \mu \mathrm{i}\left[2 p \gamma_{1} a_{1} A \mathrm{e}^{-\gamma_{1} z}+2 p \gamma_{2} a_{2} B \mathrm{e}^{-\gamma_{2} z}+\left(C p-D \gamma_{3}\right) \mathrm{e}^{-\gamma_{3} z}\right]= \\
& \sum_{m=1}^{\infty} \hat{\sigma}_{y z e m}(0) g_{\mathrm{em}}^{\left(-a_{\mathrm{e}}, a_{\mathrm{e}}\right)}(p)+\sum_{m=1}^{\infty} \hat{\sigma}_{y z \mathrm{~b}^{-} m}(0) g_{\mathrm{b}^{-} m}^{\left(-b_{2},-b_{1}\right)}(p)+\sum_{m=1}^{\infty} \hat{\sigma}_{y z \mathrm{~b}^{+} m}(0) g_{\mathrm{b}^{+} m}^{\left(b_{1}, b_{2}\right)}(p), \\
& g_{3} A \mathrm{e}^{-\gamma_{1} z}+g_{4} B \mathrm{e}^{-\gamma_{2} z}-2 \mu \gamma_{3} C \mathrm{e}^{-\gamma_{3} z}= \\
& \sum_{m=0}^{\infty} \hat{\sigma}_{z z e m}(0) f_{\mathrm{em}}^{\left(-a_{\mathrm{e}}, a_{\mathrm{e}}\right)}(p)+\sum_{m=0}^{\infty} \hat{\sigma}_{z z \mathrm{~b}^{-} m}(0) f_{\mathrm{b}^{-} m}^{\left(-b_{2},-b_{1}\right)}(p)+\sum_{m=0}^{\infty} \hat{\sigma}_{z z \mathrm{~b}^{+} m}(0) f_{\mathrm{b}^{+} m}^{\left(b_{1}, b_{2}\right)}(p),
\end{aligned}
$$

where $f_{j m}^{(a, b)}(p)$ and $g_{j m}^{(a, b)}(p)$ are defined as

$$
\begin{aligned}
& f_{j m}^{(a, b)}(p)=\int_{a}^{b} \cos \left(p_{j m} T_{j}(y)\right) \mathrm{e}^{-\mathrm{i} p y} \mathrm{~d} y, \\
& g_{j m}^{(a, b)}(p)=\int_{a}^{b} \sin \left(p_{j m} T_{j}(y)\right) \mathrm{e}^{-\mathrm{i} p y} \mathrm{~d} y .
\end{aligned}
$$

An additional equation can be obtained by assuming the drainage condition at the interface to be fully permeable, and this gives:

$$
A+B=0
$$

The unknowns $A, B, C$ and $D$ in the poroelastic half-space that depend on the continuous Fourier variable $p$ can be solved and expressed in terms of $D_{n m}^{j}$ and $E_{n m}^{j}$ (independent of $p$ ) by use of Eqs. (46)-(48) and (51).

The constants $D_{n m}^{j}$ and $E_{n m}^{j}$ remain to be solved. They can be solved by adopting the 
remaining boundary conditions in the embankment and in the right bank. Employing an inverse Fourier series with respect to $y$ to the displacement boundary condition Eq. (3) over the width of the embankment, three equations are obtained for each mode $m$, denoted with $m^{\prime}$ :

$$
\begin{aligned}
& \frac{1}{2 \pi} \int_{-\infty}^{+\infty}-\frac{\mathrm{i}}{q}\left\{\left[\chi_{1}+a_{1}\left(q^{2}-L_{1}^{2}\right)\right] A+\left[\chi_{2}+a_{2}\left(q^{2}-L_{2}^{2}\right)\right] B+\left(C \gamma_{3}+D p\right)\right\} f_{\mathrm{e} m^{\prime}}^{\prime\left(-a_{\mathrm{e}}, a_{\mathrm{e}}\right)}(p) \mathrm{d} p \\
& =\frac{2 a_{\mathrm{e}}}{\varepsilon_{m^{\prime}}} \hat{u}_{\mathrm{e} m^{\prime}}(0), \quad m^{\prime}=0,1, \ldots, \\
& \frac{1}{2 \pi} \int_{-\infty}^{+\infty}\left[-\mathrm{i} p\left(a_{1} A+a_{2} B\right)+\mathrm{i} D\right] g_{\mathrm{e} m^{\prime}}^{\prime\left(-a_{e}, a_{\mathrm{e}}\right)}(p) \mathrm{d} p=a_{\mathrm{e}} \hat{v}_{\mathrm{e} m^{\prime}}(0), \quad m^{\prime}=1,2, \ldots, \\
& \frac{1}{2 \pi} \int_{-\infty}^{+\infty}\left(\gamma_{1} a_{1} A+\gamma_{2} a_{2} B+C\right) f_{\mathrm{e} m^{\prime}}^{\prime\left(-a_{\mathrm{e}}, a_{\mathrm{e}}\right)}(p) \mathrm{d} p=\frac{2 a_{\mathrm{e}}}{\varepsilon_{m^{\prime}}} \hat{w}_{\mathrm{e} m^{\prime}}(0), \quad m^{\prime}=0,1, \ldots,
\end{aligned}
$$

where

$$
\begin{gathered}
f_{j m}^{(a, b)}(p)=\int_{a}^{b} \cos \left(p_{j m} T_{j}(y)\right) \mathrm{e}^{\mathrm{i} p y} \mathrm{~d} y, \\
g_{j m}^{\prime(a, b)}(p)=\int_{a}^{b} \sin \left(p_{j m} T_{j}(y)\right) \mathrm{e}^{\mathrm{i} p y} \mathrm{~d} y .
\end{gathered}
$$

As the series solution in the embankment layer is orthogonal over the width $2 a_{\mathrm{e}}$, each mode of the series gives a separate equation. Here $\varepsilon_{m^{\prime}}$ is the Neumann factor, defined as $\varepsilon_{0}=1$ and $\varepsilon_{m^{\prime}}=2$ for $m^{\prime} \geq 1$. A similar inverse Fourier series is employed to the displacement interface condition in the right bank $j=\mathrm{b}^{+}$( Eq. (3)). Another set of equations is obtained as:

$$
\begin{aligned}
& \frac{1}{2 \pi} \int_{-\infty}^{+\infty}-\frac{\mathrm{i}}{q}\left\{\left[\chi_{1}+a_{1}\left(q^{2}-L_{1}^{2}\right)\right] A+\left[\chi_{2}+a_{2}\left(q^{2}-L_{2}^{2}\right)\right] B+\left(C \gamma_{3}+D p\right)\right\} f_{b^{+} m^{\prime}}^{\prime\left(b_{1}, b_{2}\right)}(p) \mathrm{d} p \\
& =\frac{b_{2}-b_{1}}{\varepsilon_{m^{\prime}}} \hat{u}_{\mathrm{b}^{+} m^{\prime}}(0), \quad m^{\prime}=0,1, \ldots, \\
& \frac{1}{2 \pi} \int_{-\infty}^{+\infty}\left[-\mathrm{i} p\left(a_{1} A+a_{2} B\right)+\mathrm{i} D\right] g_{\mathrm{b}^{+} m^{\prime}}^{\prime\left(b_{1}, b_{2}\right)}(p) \mathrm{d} p=\frac{b_{2}-b_{1}}{2} \hat{v}_{\mathrm{b}^{+} m^{\prime}}(0), \quad m^{\prime}=1,2, \ldots, \\
& \frac{1}{2 \pi} \int_{-\infty}^{+\infty}\left(\gamma_{1} a_{1} A+\gamma_{2} a_{2} B+C\right) f_{\mathrm{b}^{+} m^{\prime}}^{\prime\left(b_{1}, b_{2}\right)}(p) \mathrm{d} p=\frac{b_{2}-b_{1}}{\varepsilon_{m^{\prime}}} \hat{w}_{\mathrm{b}^{+} m^{\prime}}(0), \quad m^{\prime}=0,1, \ldots .
\end{aligned}
$$

A similar inverse Fourier series is applied over the width $2 a_{\mathrm{e}}$ on the conditions for the tractions at 
the top surface of the embankment (Eq. (7-9)). This gives the following equations:

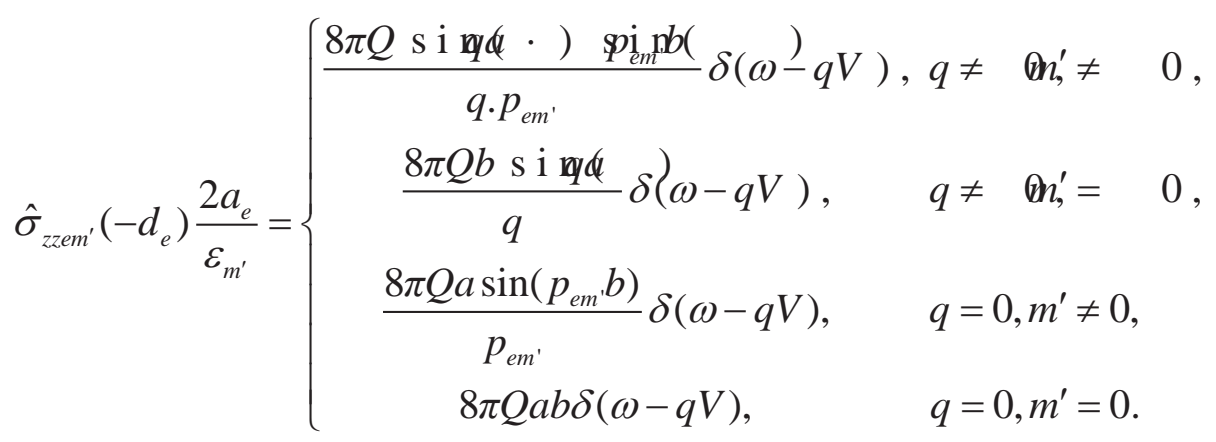

$$
\begin{aligned}
& \hat{\sigma}_{y z m^{\prime}}\left(-d_{\mathrm{e}}\right)=0, \quad m^{\prime}=1,2 \ldots, \\
& \hat{\sigma}_{x z e m^{\prime}}\left(-d_{\mathrm{e}}\right)=0, \quad m^{\prime}=0,1 \ldots,
\end{aligned}
$$

$a$ and $b$ are the half width and length of the rectangular load, respectively.

The last set of equations is obtained from the traction-free surface of the right bank (Eq. (10)):

$$
\begin{array}{ll}
\hat{\sigma}_{z z b^{+} m^{\prime}}\left(-d_{b}\right)=0, & m^{\prime}=0,1, \ldots, \\
\hat{\sigma}_{y z b^{+} m^{\prime}}\left(-d_{b}\right)=0, & m^{\prime}=1,2, \ldots, \\
\hat{\sigma}_{x z b^{+} m^{\prime}}\left(-d_{b}\right)=0, & m^{\prime}=0,1, \ldots .
\end{array}
$$

The obtained equations, (52-54) and (57)-(65), are finally used to obtain the solution for the constants $D_{n m}^{j}$ and $E_{n m}^{j}$. Then, by substituting $D_{n m}^{j}$ and $E_{n m}^{j}$ into Eqs.(46)-(48), the coefficients $A, B, C$ and $D$ in the poroelastic half-space can be obtained. In this way, the dynamic responses to the moving load are determined at all positions.

\section{Illustrations and results of analyses}

\subsection{Validation of the methodology}

The parameters for the elastic layers are listed in Table 3, which are selected corresponding to those used in Karlström and Boström [19]. A saturated poroelastic half-space is specified by the parameters in Table 4 referring to Theodorakopoulos [23]. The parameters are chosen according to Tables 3-4 if not otherwise stated in the figures. The amplitude of the load pressure is chosen as 25 
$\mathrm{kN} / \mathrm{m}^{2}$. The value of $b_{2}$ is chosen as $50 \mathrm{~m}$ by a trial calculation for satisfactory precision, and the number of terms $(m)$ are chosen linearly proportional to the widths (in meters) of the rectangular layers by the constant of proportionality 0.75 according to Karlström and Boström [19]. The computing time is within hours for one curve. The case of an ideal elastic half-space soil medium can be simulated by choosing negligibly small values for the poroelastic parameters $\left(\rho_{\mathrm{f}}, m_{1}, M\right.$, $\frac{\rho_{\mathrm{f}} g}{k_{\mathrm{d}}}$ and $\left.\alpha\right)$

To validate the accuracy of the current method, results obtained from the presented methodology are compared with those available in Dasgupta et al. [9]. In their work, the screening effects of a single open trench to a foundation generated by a harmonic point load with excitation frequency $50 \mathrm{~Hz}$ were studied, neglecting the mass of the foundation. By replacing the foundation with an equivalent rectangular pressure, the same problem is investigated by the present methodology. The poroelastic half-space is degenerated into an elastic half-space and the same parameters are chosen as those used in Dasgupta et al. [9]. The normalized vertical displacements, defined as the ratio of the displacement in the presence of the trench over that without trench, are presented in Fig. 2. It can be seen that the general trend of the two results are in good agreement, but also some discrepancy can be observed at the edge of the trench, this may be due to the effect of the artificial boundary condition $\left(v_{\mathrm{e}}=0\right)$ used in the present model for the load excitation frequency $50 \mathrm{~Hz}$. It is also of interest to note that the ground frequency components generated by a moving constant load usually lie in a lower frequency range, below $20 \mathrm{~Hz}$.

\subsection{Screening efficiency of trenches}

In Fig.3, the ground surface velocity responses are presented against $x$ both in front of and outside the trench for load speeds $20 \mathrm{~m} / \mathrm{s}$ and $60 \mathrm{~m} / \mathrm{s}$. Two distances from the load are selected and 
shown in the figure as $y=5 \mathrm{~m}$ in front of the trench and $y=10 \mathrm{~m}$ outside the trench. It can be seen at $y=5 \mathrm{~m}$ that no screening effect can be observed, and the velocity responses for the trench model are even larger than that for the model without trench. At $y=10 \mathrm{~m}$ outside the trench, some screening can be observed for the load speed $20 \mathrm{~m} / \mathrm{s}$ and the velocity responses are quite small at this load speed. But as the load speed exceeds the critical speed, in this case to $60 \mathrm{~m} / \mathrm{s}$, the maximum velocity responses become much larger and fluctuate fiercely along the $x$ axis. The critical speed herein is defined as the Rayleigh wave speed of the ground surface, about $56 \mathrm{~m} / \mathrm{s}$. The screening effectiveness of the trench becomes more significant as the load speed increases.

In Fig. 4, the excessive pore water pressures in the ground are presented at the depth $1 \mathrm{~m}$ under the ground water table. It can be observed that the maximum positive pore water pressure increases both at $y=5 \mathrm{~m}$ and $y=10 \mathrm{~m}$ after the installation of the trench. This means that the open trench aggravate the pore water pressure response in the saturated soil medium in front of and outside the trench. The cause of this phenomenon may due to that the waves propagating in the top elastic layer scatter at the sides and bottom of the trenches, which leads to an increase of the portion of energy passing beneath the trench. Thus, the excessive pore water pressure responses under the trench increase. It is also of interest to note that the amplitude of the negative pore water pressure for the trench model is much larger than that for the model without trench. The increase of the negative pore water pressure will result in an increase of the effective stresses in the saturated soil medium after the moving load passed by.

In the following paragraph, the screening effect of the open trench will be evaluated using the reduction of vibration level in terms of decibels, i.e.,

Reduction of vibration level $A_{\mathrm{r}}[\mathrm{dB}]=-20 \log \left(\mathrm{P}_{1} / \mathrm{P}_{2}\right)$, 
where $\mathrm{P}_{1}$ is the computed maximum velocity responses at a certain place in presence of trenches and $\mathrm{P}_{2}$ is the reference maximum velocity responses at the same place obtained from an associated analysis without trench.

In Fig. 5, the reduction of vibration levels against $y$ are presented for both sub- and supercritical load speed. A high amplification of the vibration can be observed at the edge in front of the trench and small reduction effectiveness can be observed in an area in front of the trench. This phenomenon is due to the constructive or destructive interference between the propagating waves and the reflected waves from the trench edge. For the load speed $20 \mathrm{~m} / \mathrm{s}$, as shown in Fig. 5(a), the reduction of vibration level at the edge outside the trench can reach the value $6 \mathrm{~dB}$, but as the distance increases, the screening effectiveness decreases. At the distance $y=20 \mathrm{~m}$, almost no isolation effect can be observed. For the supercritical load speed $60 \mathrm{~m} / \mathrm{s}$, as shown in Fig. 5(b), the screening efficiency of the trench has increased. At the edge outside the trench, the reduction of vibration levels can reach $10 \mathrm{~dB}$. The reduction of vibration levels also decreases with increasing distance for this load speed; but a reduction of vibration level $2.5 \mathrm{~dB}$ is still obtained at $y=30 \mathrm{~m}$. A similar phenomenon that the screening effects decrease with increase of $y$ is also found in With et al. [4] and validated by field measurement results. The results for the trench on the single-phase elastic half-space are presented in Fig. 5 for comparison. It is clearly visualized from Fig. 5 that, for the load speed $20 \mathrm{~m} / \mathrm{s}$, the screening effectiveness of the trench on the poroelastic half-space is close to that of the trench on the elastic half-space; but for the load speed $60 \mathrm{~m} / \mathrm{s}$, the discrepancy of the screening efficiency between the two models is significant. The reduction of vibration levels of the trench on the elastic half-space is about $1 \mathrm{~dB}$ smaller than that of the trench on the poroelastic half-space. 
3.3 Parametric study: Influence of load speed, soil permeability and the distance of trenches to load

In order to represent the reduction effect over an area behind the trench, an average reduction level $\overline{A_{\mathrm{r}}}$ over a distance $T$ behind the trench is computed by:

$$
\overline{A_{\mathrm{r}}}=\frac{1}{T} \int_{b_{1}}^{b_{1}+T} A_{\mathrm{r}} d y
$$

where $T$ is chosen as $20 \mathrm{~m}$. In Fig. 6, the average reduction of velocity level is presented against the dimensionless load speed $c_{0}$, which is defined as $V / c_{\mathrm{s}}$, where $c_{\mathrm{S}}$ is the shear wave velocity of the half-space. It can be observed that the average reduction of velocity level is about $1.5 \mathrm{~dB}$ at $c_{0}=0.4$ and has a slight increase as the dimensionless load speed increases from 0.4 to 0.7 . On the other hand, a rapid increase of the reduction level can be observed as the load speed increases from 0.8 to 1.0; this means that the open trench becomes more efficient in mitigating the ground vibrations as the load speed approaches or exceeds the critical speed. Further increase of the load speed leads to a small increase of the average reduction level. It can also be clearly observed that the discrepancy of the screening efficiency between the trenches on the poroelastic half-space and elastic half-space becomes more significant as the load speed increases; the discrepancy between the two models is about $1 \mathrm{~dB}$ for load speeds near and above the critical wave speed. Thus, the use of the single-phase elastic soil model will underestimate the screening efficiency of the trench significantly when the load speed approaches or exceeds the critical speed.

In Fig. 7, the effects of the permeability coefficients $k_{\mathrm{d}}$ of the saturated poroelastic half-space on the reduction velocity level are studied at the load speed $60 \mathrm{~m} / \mathrm{s}$. Four values of $k_{\mathrm{d}}$ are shown. It is noted in Fig. 7 that the permeability of the soil has a small effect on the reduction velocity level, and the reduction velocity level decreases as $k_{\mathrm{d}}$ increases for various distances behind the trench. This phenomenon is due to the fact that, as the permeability coefficients $k_{\mathrm{d}}$ increases, the coupling 
effect between the soil skeleton and fluid becomes weaker. Thus, attenuation effects of the saturated soil medium to the waves reduce, which leads to a reduction of the screening effect of the trench.

In Fig. 8, the effects of $L$ on the screening efficiency of the trench are investigated. $L$ is defined as the distance of the trench from the load center. The geometry of the trench is kept unchanged for various $L$. It is observed that the screening effectiveness of the trench is much improved as $L$ increases, especially at the area near behind the trenches. That is to say, the same trench located farther from the load, the better isolation effects can be achieved at the place behind the trench. The same phenomenon is also observed by Yang and Hung [5]. Meanwhile, the vibration amplification effect in front of the trenches becomes weak as $L$ increases.

To further explore the effect of $L$ on the trench's screening efficiency, the average reduction level for different $L$ is presented in Fig. 9. The average reduction level is calculated within the area $20 \mathrm{~m}$ behind the trench. It can be seen that, as $L$ increases from $6 \mathrm{~m}$ to $16 \mathrm{~m}$, the average reduction velocity level increases from $3.2 \mathrm{~dB}$ to $12 \mathrm{~dB}$ for the trench on the poroelastic half-space. Thus, in order to obtain a better screening effect and less detrimental amplification in front of the trench, the trenches are suggested to be installed as close as possible to the concerned structure. In addition, it is noted that the average reduction velocity level of the trench on the elastic half-space is about $1 \mathrm{~dB}$ smaller than that of the trench on the poroelastic half-space for various $L$.

\section{Conclusions}

In this paper, an analytical model is proposed to investigate the screening efficiency of trenches to moving-load induced ground vibrations. A fully saturated poroelastic half-space soil model is utilized and Biot's fully dynamic poroelastic theory is applied to characterize the saturated 
poroelastic half-space, considering the coupling effect between the soil skeleton and the fluid. The screening effects of open trenches to ground surface vibration velocities and excessive pore water pressures in the saturated soil medium are investigated. The different performances of the trenches on the single-phase elastic half-space and the poroelastic half-space are especially identified. Furthermore, the effects of the distance between the trench and the load on the screening efficiency are analyzed.

The main conclusions of this study are summarized below:

1. Amplifications of the ground surface vibrations can be observed in front of the trenches, while apparent screening effects can be obtained outside the trench; the screening effect of the trench to the ground surface vibrations improves as the load speed increases. The installation of the trench aggravates the excesses pore water pressure responses in the underlying saturated soil medium both before and behind the trench, especially the negative pore water pressure responses after the load has passed by.

2. The discrepancy between the screening efficiency of trench on a poroelastic soil and that of trench on an elastic soil model becomes apparent as the load speed increases. The use of the single-phase elastic soil model will underestimate the screening efficiency of the trench significantly when the load speed approaches or exceeds the critical speed. The permeability coefficient of the saturated soil medium has an apparent effect on the trench's screening efficiency when the load speed is high.

3. The distance between the trench and the moving load is an important factor for the trench design. The screening effectiveness behind the trench can be much improved when the distance of the trench from the moving load increases. Meanwhile, the detrimental amplification of vibrations 
in front of the trench can be alleviated if the distance of the trench from the moving load increases.

\section{Acknowledgement}

This work is part of the activities of the Center of Excellence CHARMEC (CHAlmers Railway MEChanics, www.charmec.chalmers.se). Zhigang Cao's one-year visit to Chalmers University of Technology as a joint-trained PhD student was supported by the China Scholarship Council and the National Natural Science Foundation of China (51025827).

\section{Appendix}

$$
\begin{gathered}
\gamma_{i}=\sqrt{q^{2}+p^{2}-L_{i}^{2}}, \quad i=1,2 \\
\gamma_{3}=\sqrt{q^{2}+p^{2}+S^{2} / \mu} \\
L_{1}^{2}=\frac{\beta_{1}+\sqrt{\beta_{1}^{2}-4 \beta_{2}}}{2}, L_{2}^{2}=\frac{\beta_{1}-\sqrt{\beta_{1}^{2}-4 \beta_{2}}}{2} \\
\beta_{1}=\frac{\left(\lambda+2 \mu+\alpha^{2} M\right)\left(m_{1} \omega^{2}-\mathrm{i} b \omega\right)-2 \alpha M \rho_{\mathrm{f}} \omega^{2}+\rho M \omega^{2}}{(\lambda+2 \mu) M} \\
\beta_{2}=\frac{\left(m_{1} \omega^{2}-\mathrm{i} b \omega\right) \rho \omega^{2}-\rho_{\mathrm{f}}^{2} \omega^{4}}{(\lambda+2 \mu) M} \\
\chi_{i}=\frac{\vartheta M L_{i}^{2}-\rho_{\mathrm{f}} \omega^{2}}{\rho_{\mathrm{f}} \omega^{2}(\alpha-\vartheta) M} \quad i=1,2 \\
g_{i}=\frac{(\lambda+\mu) \chi_{i}-\alpha+\vartheta}{S^{2}-\mu L_{i}^{2}} \quad i=1,2 \\
\vartheta=\rho_{\mathrm{f}} \omega^{2} /\left(m_{1} \omega^{2}-\mathrm{i} b \omega\right)
\end{gathered}
$$




$$
g_{3}=\lambda \chi_{1}-2 \mu \gamma_{1}^{2} a_{1}-\alpha, g_{4}=\lambda \chi_{2}-2 \mu \gamma_{2}^{2} a_{2}-\alpha
$$

\section{References}

[1] G. Segol, P.C.Y. Lee, J.F. Abel, Amplitude reduction of surface waves by trenches, Journal of Engineering Mechanics Division 104(3) (1978) 621-641.

[2] W.A. Haupt, Isolation of vibration by concrete core walls. Proceedings of the 9th International Conference on Soil Mechanics and Foundation Engineering, Vol. 2, Japanese Society of Soil Mechanics and Foundation Engineering, Tokyo, Japan, 1977, pp. 251-256.

[3] H. Takemiya, Field vibration mitigation by honeycomb WIB for pile foundations of a highspeed train viaduct, Soil Dynamics and Earthquake Engineering 24 (2004) 69-87.

[4] C. With, M. Bahrekazemi, A. Bodare, Wave barrier of lime-cement columns against train-induced ground-borne vibrations, Soil Dynamics and Earthquake Engineering 29 (2009) 1027-1033.

[5] Y. YANG, H. HUNG, A parametric study of wave barriers for reduction of train-induced vibrations, International Journal For Numerical and Analytical Methods in Geomechanics 40 (1997) 3729-3747.

[6] H.H. Hung, Y. B. Yang, D.W. Chang, Wave Barriers for reduction of train-induced vibrations in soils, Journal of Geotechnical and Geoenvironmental Engineering 130(12) (2004) 1283-1291.

[7] K. Emad, G.D. Manolis, Shallow trenches and propagation of surface waves, Journal of Engineering Mechanics 111(2) (1985) 279-282.

[8] D.E. Beskos, B. Dasgupta, I.G. Vardoulakis, Vibration isolation using open or filled trenches. Part1: 2-D homogeneous soil, Computational Mechanics 1(1986) 43-63. 
[9] B. Dasgupta, D.E. Beskos, I.G. Vardoulakis, Vibration isolation using open or filled trenches. Part2: 3-D homogeneous soil, Computational Mechanics 6(1990) 129-142.

[10] S. Ahmad, T.M. Al-Hussauni, Simplified design for vibration screening by open and in-filled trenches, Journal of Geotechnical Engineering 177(1) (1991) 67-88.

[11] R. Klein, H. Antes, D. Le Houedec, Efficient 3D modeling of vibration isolation by open trenches, Computer \& Structures 64(1997) 809-17.

[12] R.D. Woods, Screening of surface waves in soils, Journal of Engineering Mechanics Division 94(SM4) (1968) 951-979.

[13] S. Liao, D.A. Sangrey, Use of piles as isolation barriers, Proceeding ASCE Journal of Geotechnical Engineering Division 104(GT9) (1978) 1139-1152.

[14] J. Avilés, F.J. Sánchez-Sesma. Foundation isolation from vibration using piles as barriers, Journal of Engineering Mechanics 114(11) (1988) 1854-1870.

[15] B. Boroomand, A.M. Kaynia, Stiffness and damping of closely spaced pile groups, First International Conference on Soil Dynamics and Earthquake Engineering, V. Karlsruhe, Germany, 1991a, pp. 490-501.

[16] B. Boroomand, A.M. Kaynia, Vibration isolation by an array of piles, First International Conference on Soil Dynamics and Earthquake Engineering, V. Karlsruhe, Germany, 1991b, pp. 683-691.

[17] Y.Q. Cai, G.Y. Ding, C.J. Xu, J. Wang, Vertical amplitude reduction of Rayleigh waves by a row of piles in a poroelastic half-space, International Journal For Numerical and Analytical Methods in Geomechanics 33 (2009) 1799-1821.

[18] B. Xu, J.F. Lu, J.H. Wang, Numerical analysis of isolation of the vibration due to moving loads 
using pile rows embedded in a poroelastic half space, Journal of Sound and Vibration 319 (2009) 940-962.

[19] A. Karlström, A. Boström, Efficiency of trenches along railways for trains moving at sub- or supersonic speeds, Soil Dynamics and Earthquake Engineering 27(2007) 625-641.

[20] A. Karlström, A. Boström, An analytical model for train-induced ground vibrations from railways, Journal of Sound and Vibration 292 (2006) 221-241.

[21] M.A. Biot, Theory of propagation of elastic waves in a fluid-saturated porous solid. Part I: low-frequency range; Part II: high-frequency range, Journal of Acoustic Society of America 28 (1956) 168-191.

[22] Y. Cai, Z. Cao, H. Sun, C. Xu, Dynamic Response of pavements on poroelastic half-space soil medium to a moving traffic load, Computers and Geotechnics 36 (2009) 52-60.

[23] D.D. Theodorakopoulos, Dynamic analysis of a poroelastic half-plane soil medium under moving loads, Soil dynamics and Earthquake Engineering 23 (2003) 521-533. 


\section{List of Figures}

Figure 1: Details of the theoretical model

Figure 2: Comparison with existing work

Figure 3: The ground surface velocity responses for two load speeds: (a) $V=20 \mathrm{~m} / \mathrm{s}$, (b) $V=60 \mathrm{~m} / \mathrm{s}$

Figure 4: The excess pore water pressure responses for two load speeds: (a) $V=20 \mathrm{~m} / \mathrm{s}$, (b) $V=60 \mathrm{~m} / \mathrm{s}$

Figure 5: The reduction velocity level against $y$ for two load speeds: (a)20m/s, (b)60m/s

Figure 6: The average reduction of velocity level against the load speed

Figure 7: The reduction velocity level against $y$ for different $k_{\mathrm{d}}$ at load speed $60 \mathrm{~m} / \mathrm{s}$

Figure 8: The reduction velocity level against $y$ for different $L$ at load speed $60 \mathrm{~m} / \mathrm{s}$

Figure 9: The average reduction of velocity level for different $L$ at load speed $60 \mathrm{~m} / \mathrm{s}$ 
Fig.1

Click here to download high resolution image

Fig.1

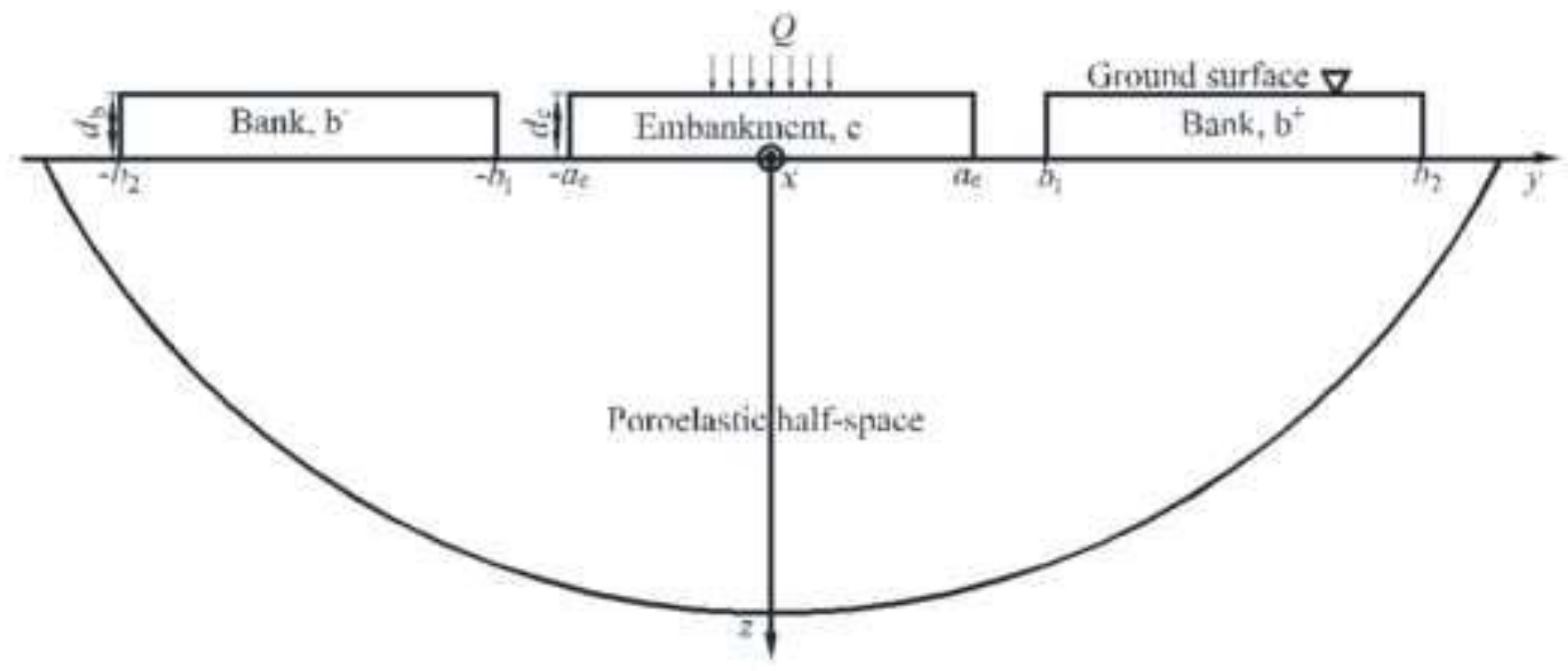

(a)

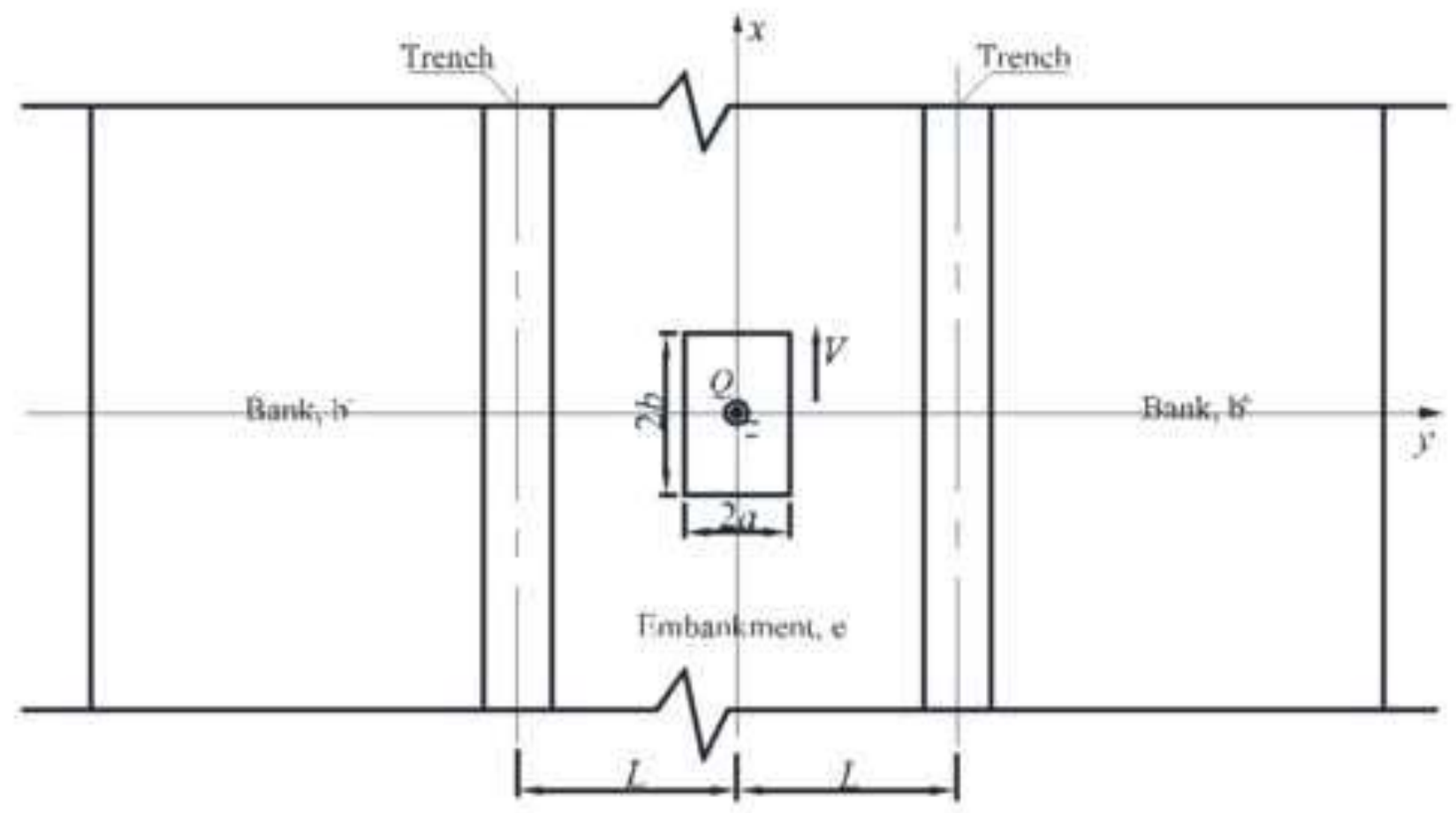

(b) 
in

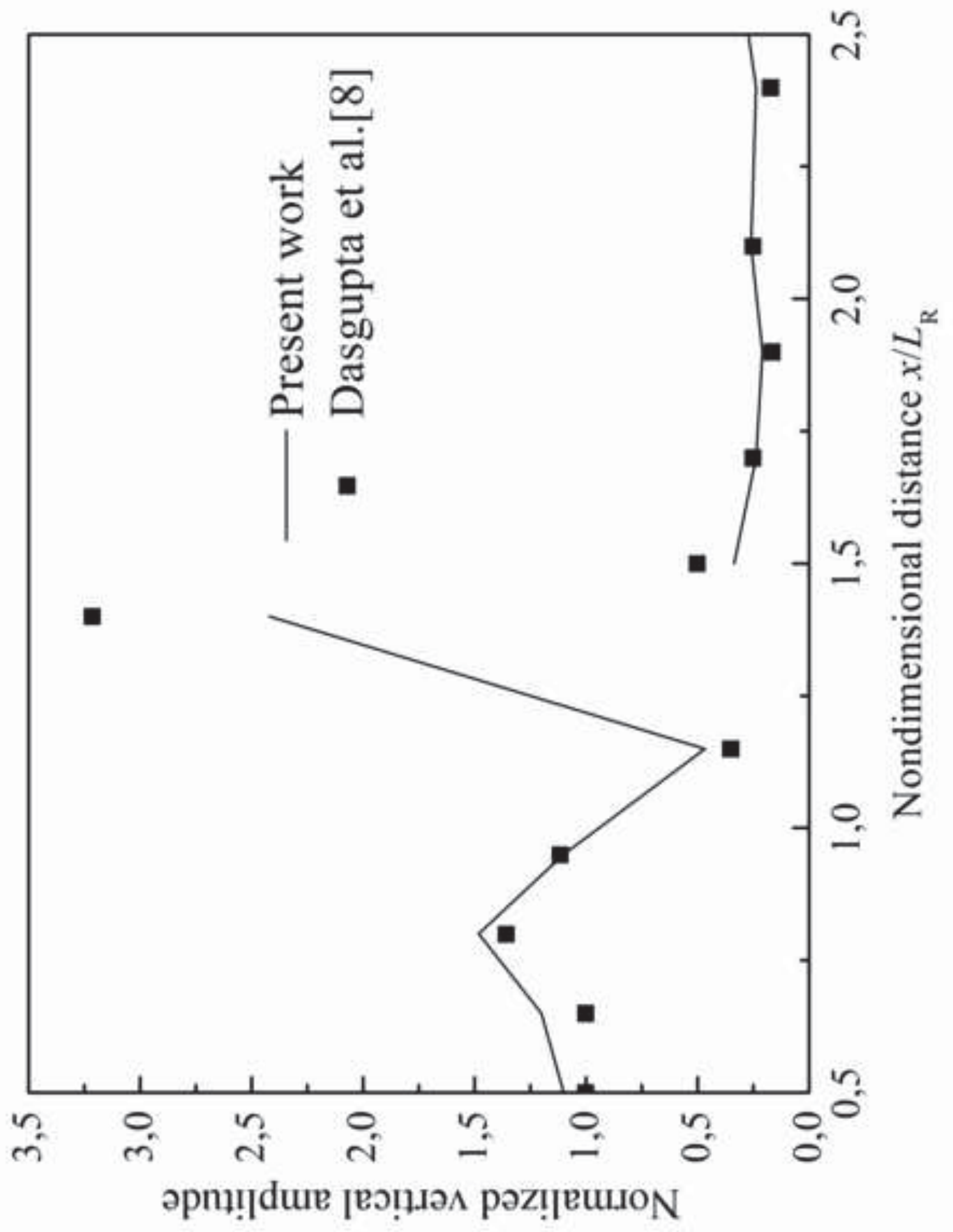

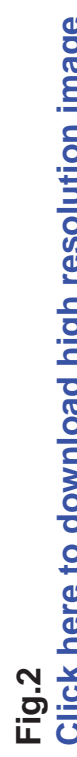


Fig.3

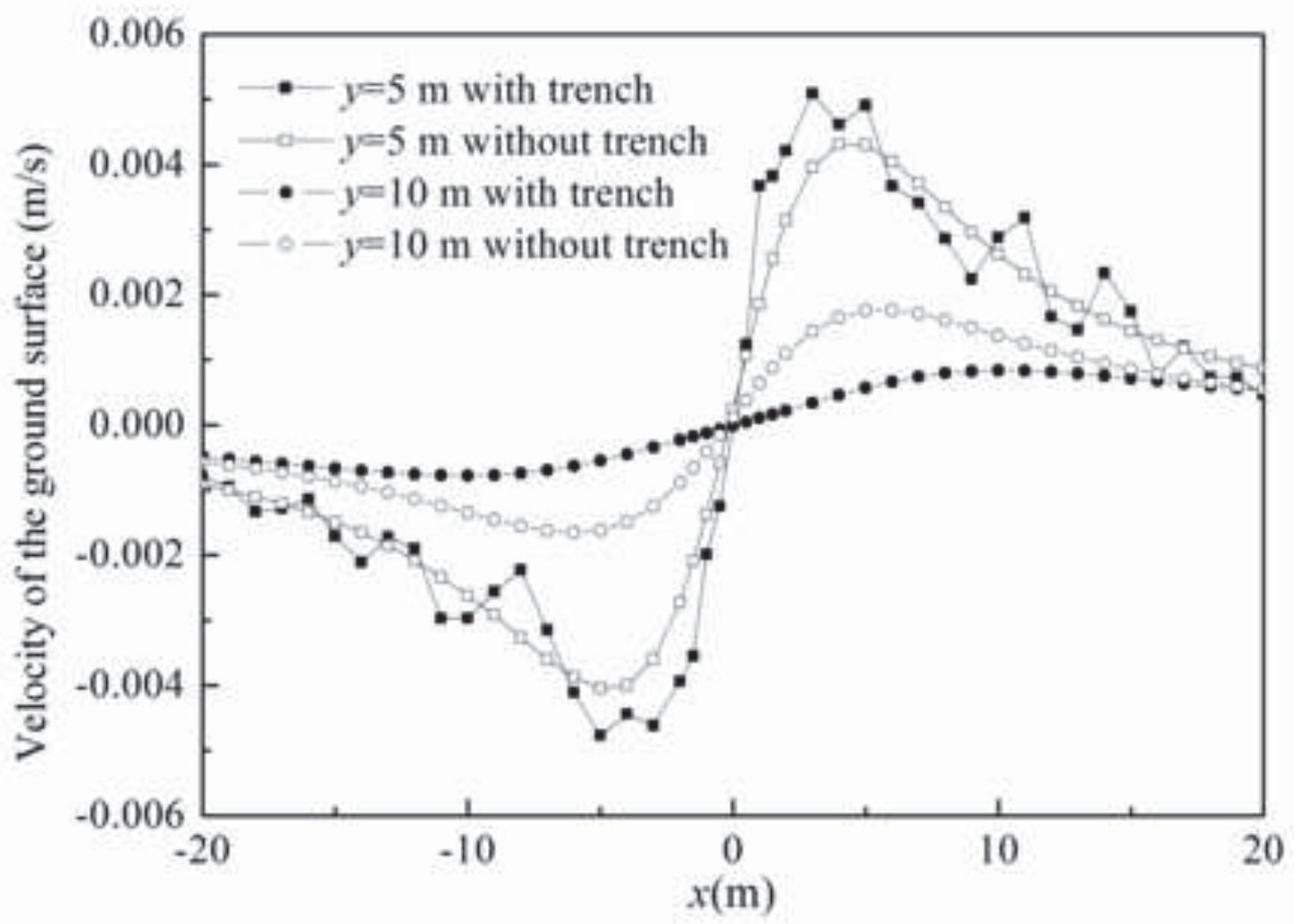

(a)

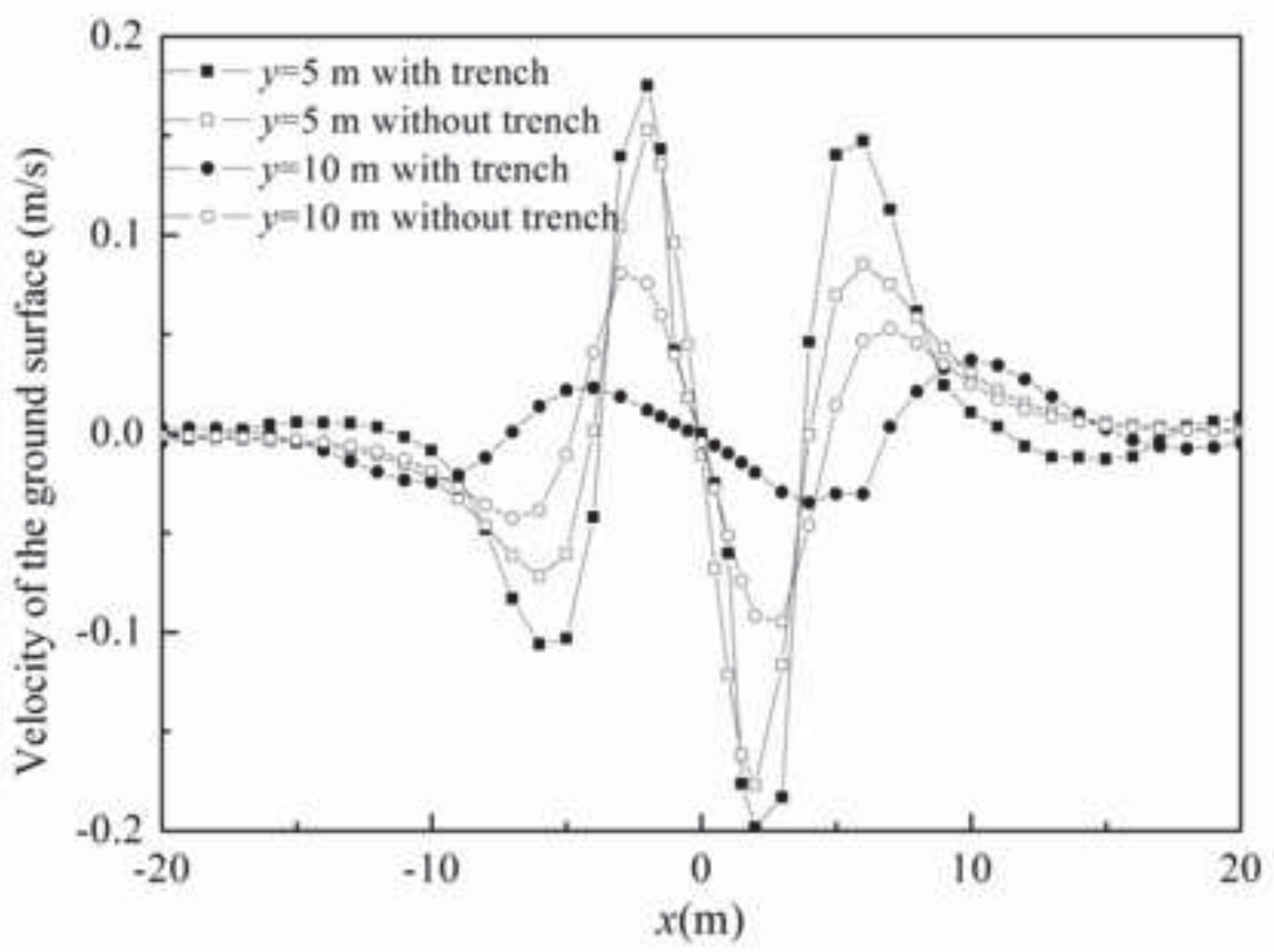

(b) 


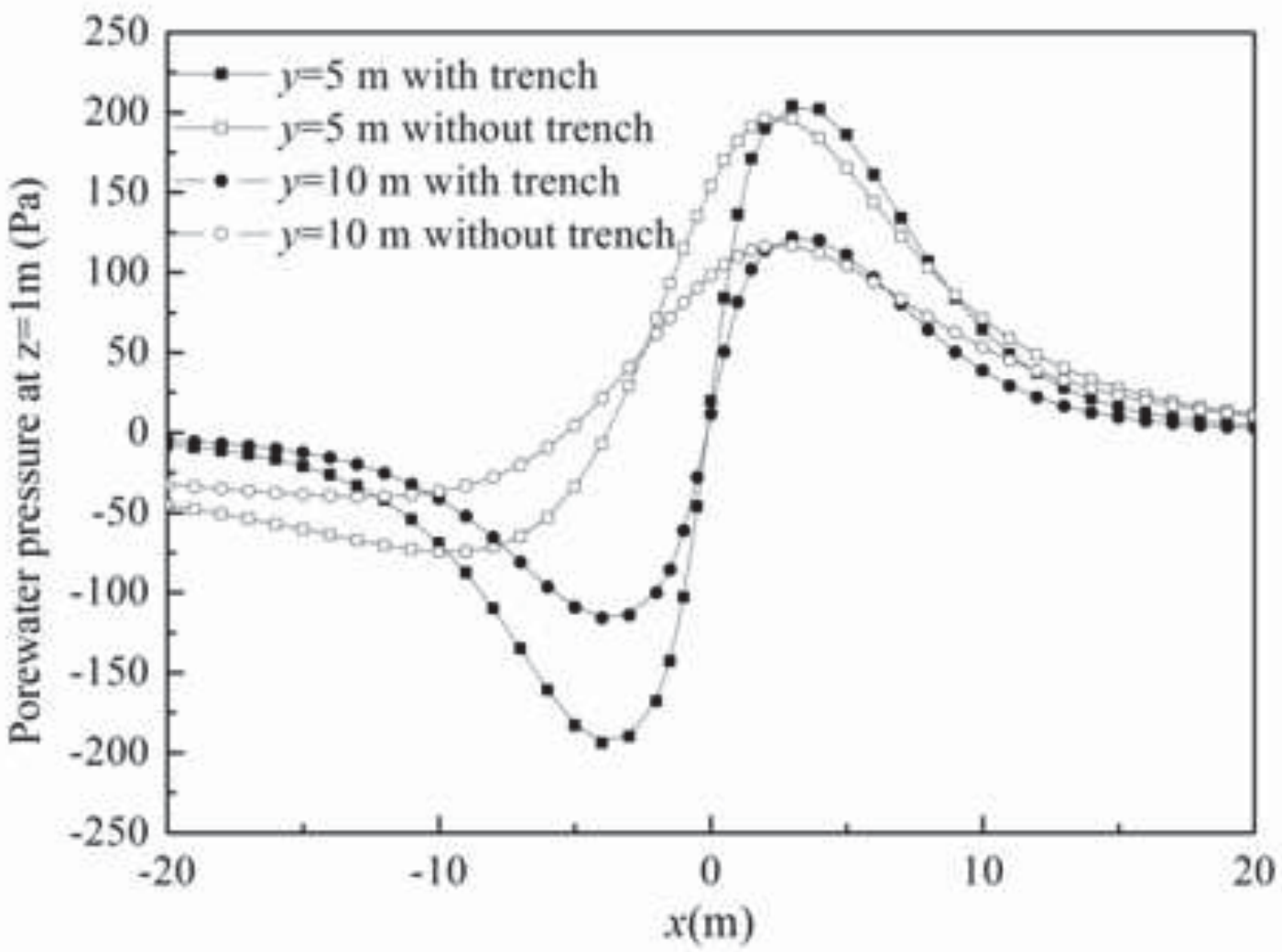

(a)

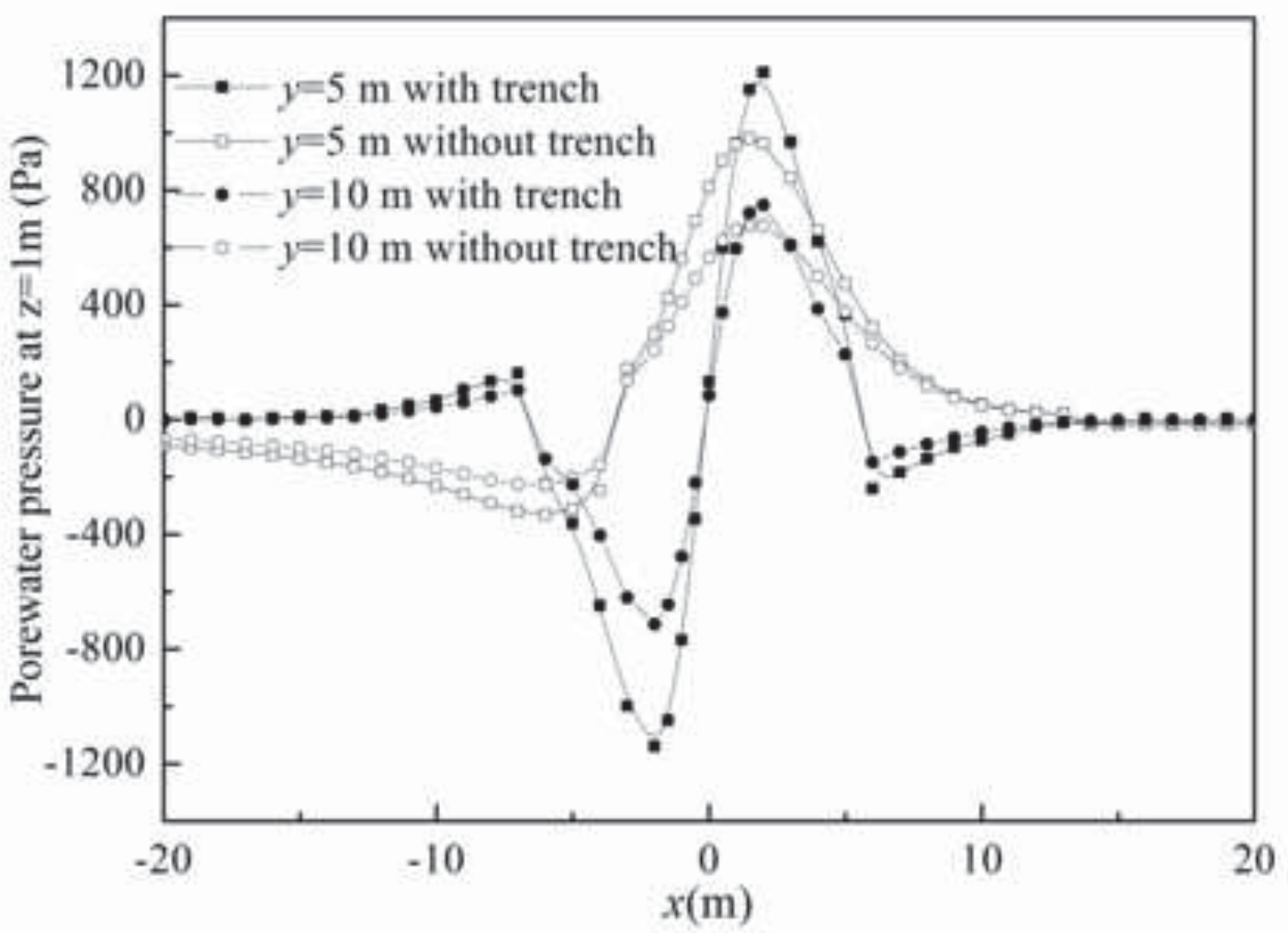

(b) 
Fig.5

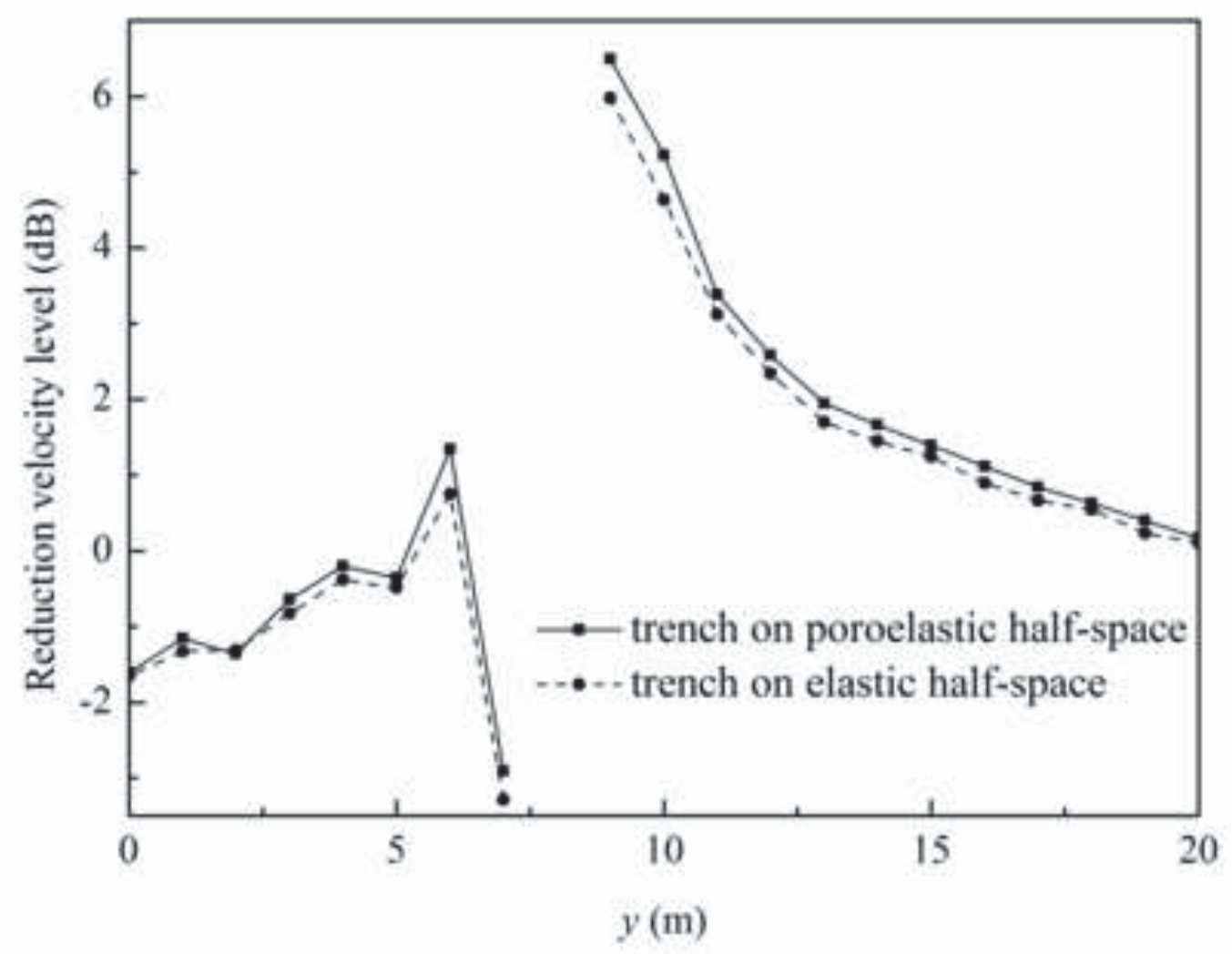

(a)

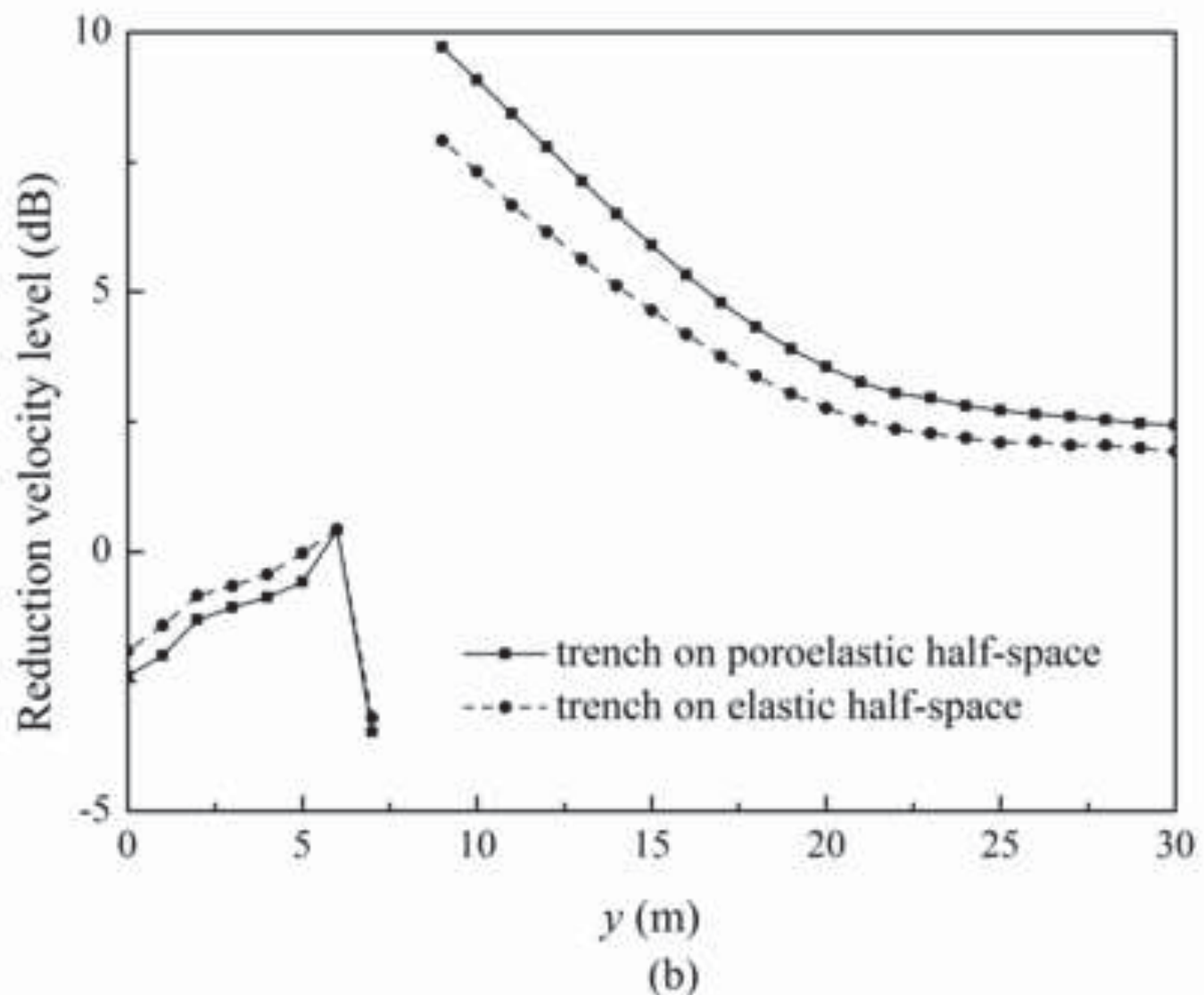


is

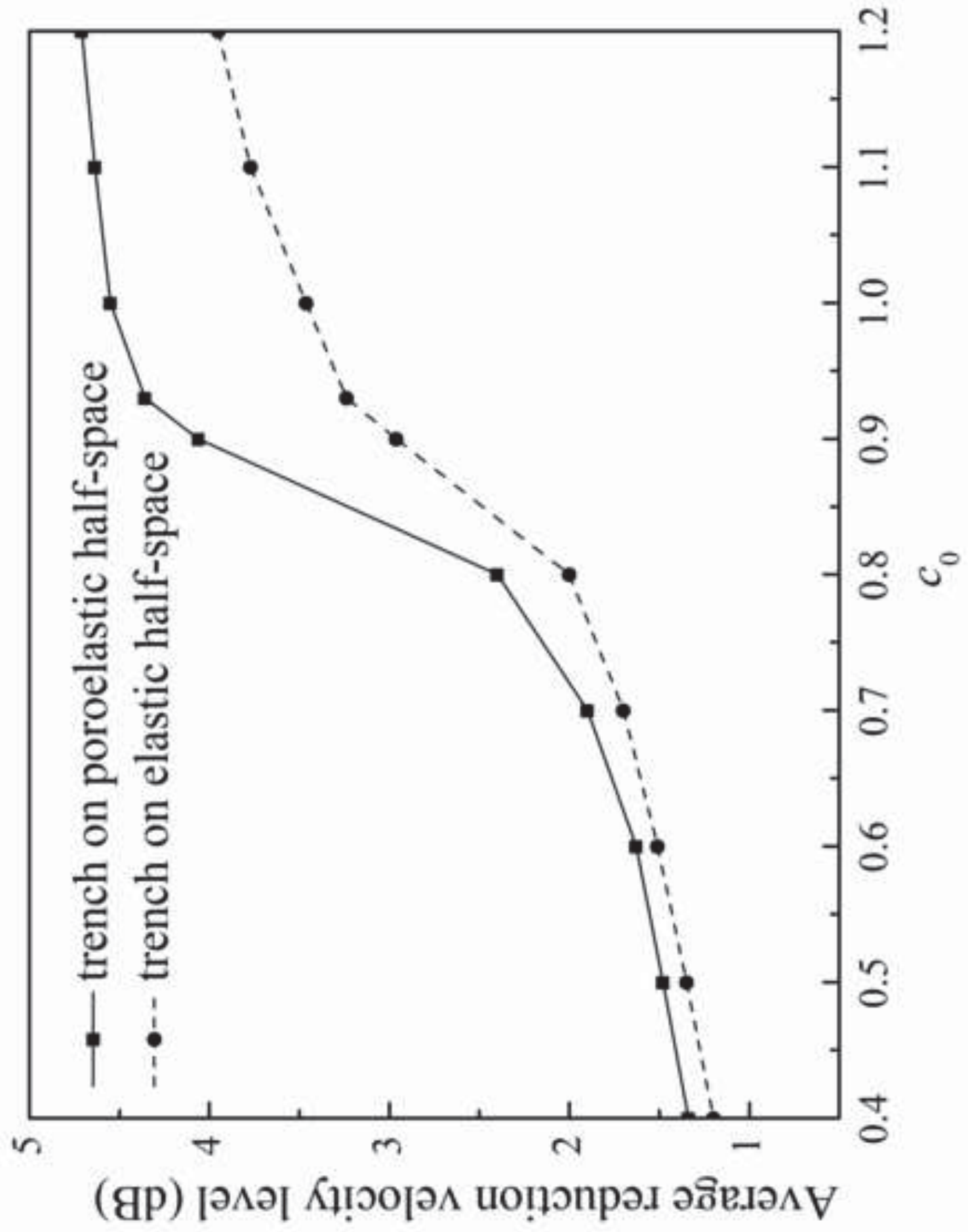


N

bi

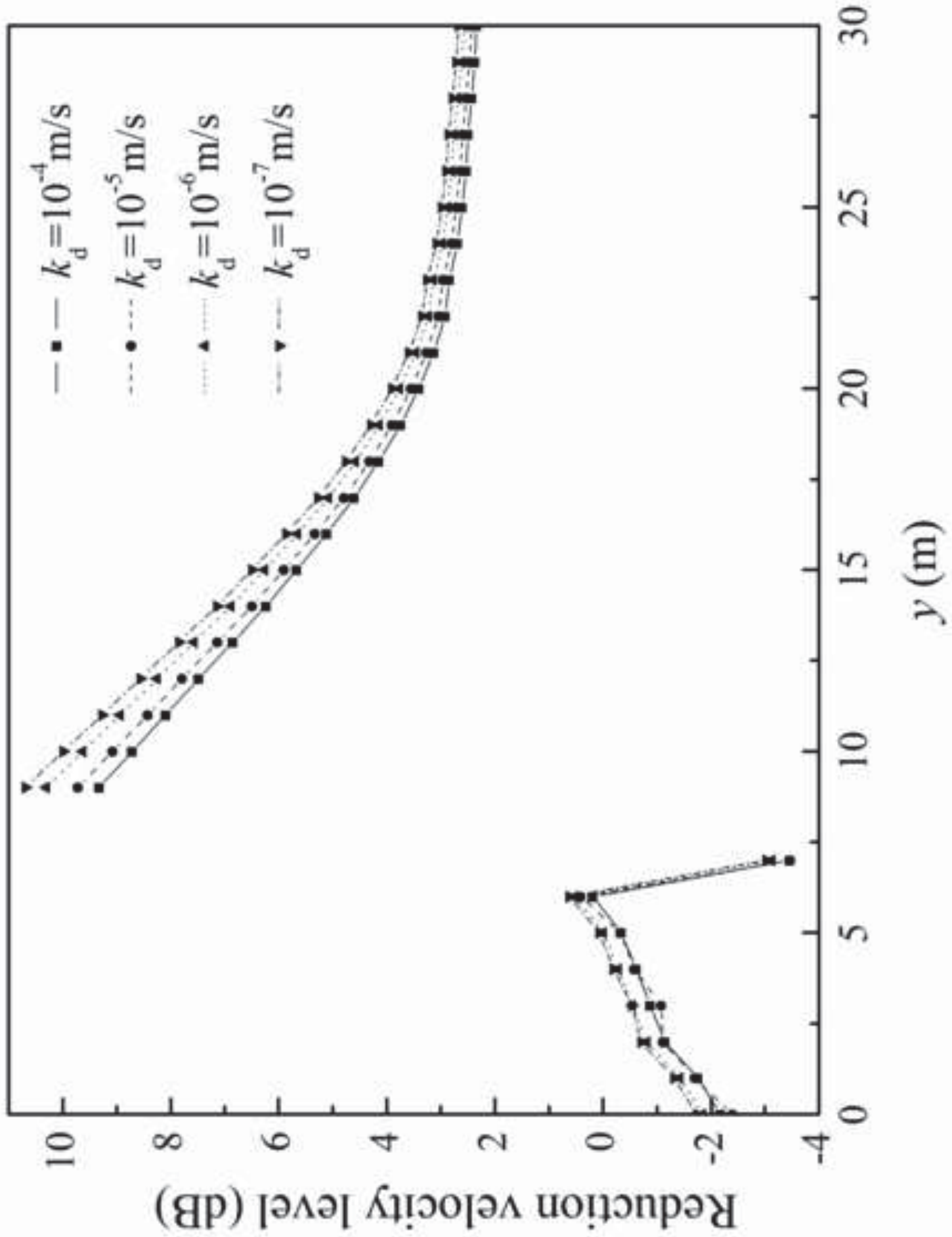

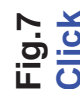


$\stackrel{\infty}{\infty}$

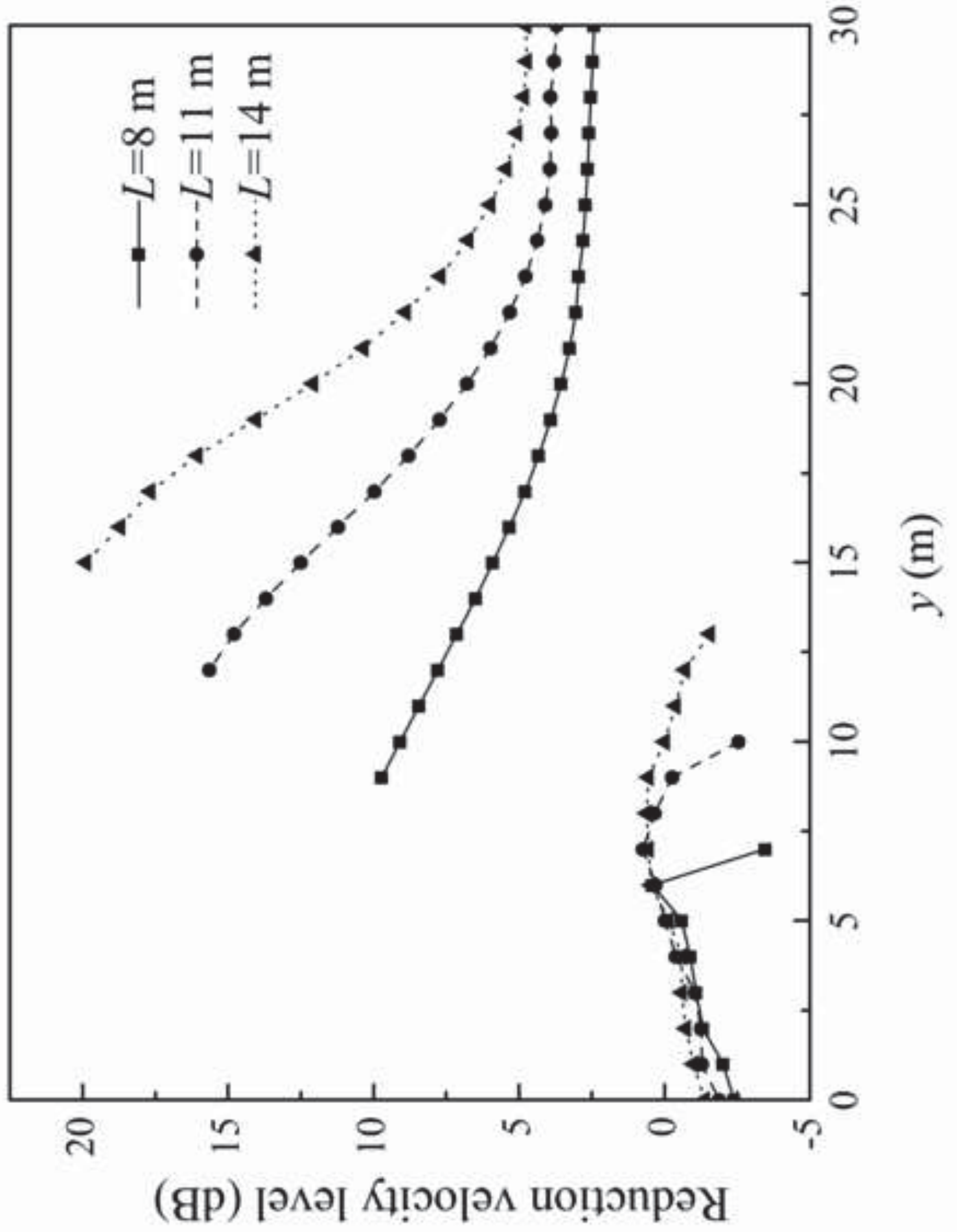

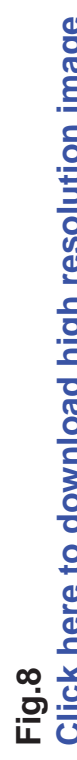


in

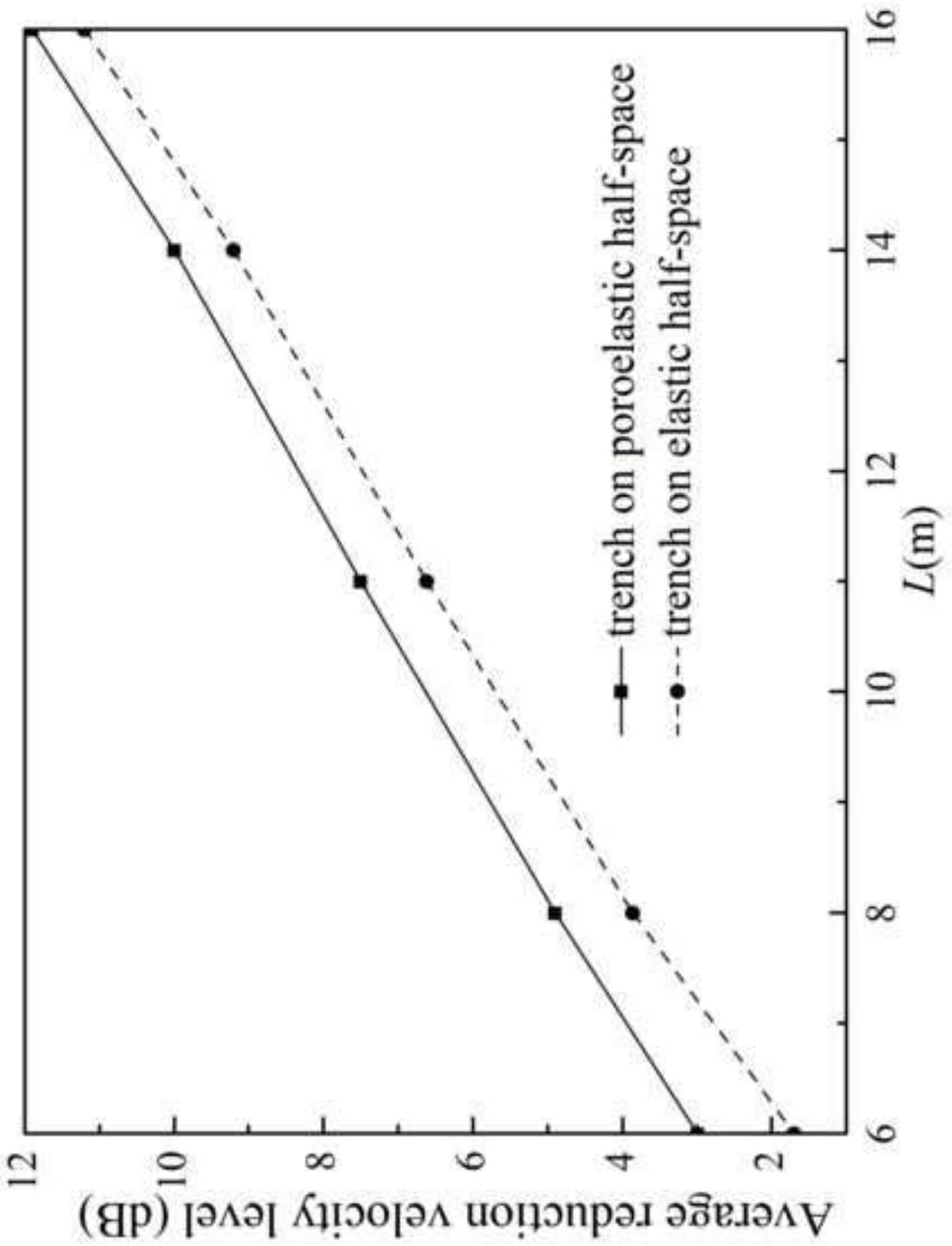

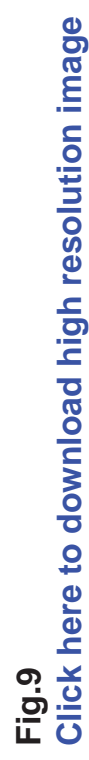


Table 1.Wave number in $y$ direction and trigonometric arguments used in the embankment layer $j=$ e and banks $j=b^{ \pm}$.

\begin{tabular}{cccc}
\hline$j$ & $\mathrm{e}$ & $\mathrm{b}^{-}$ & $\mathrm{b}^{+}$ \\
$p_{j m}$ & $\frac{m \pi}{a_{\mathrm{e}}}$ & $\frac{m \pi}{b_{1}-b_{2}}$ & $\frac{m \pi}{b_{2}-b_{1}}$ \\
$T_{j}(y)$ & $y$ & $y+b_{1}$ & $y-b_{1}$ \\
\hline
\end{tabular}


Table 2. Displacement coefficients

\begin{tabular}{cccc}
\hline$n=$ & 1 & 2 & 3 \\
$\alpha_{n m}^{j}$ & $\mathrm{i} q$ & $p_{j m}$ & $\mathrm{i} q h_{j m}^{\mathrm{S}}$ \\
$\beta_{n m}^{j}$ & $-p_{j m}$ & $-\mathrm{i} q$ & $-p_{j m} h_{j m}^{\mathrm{S}}$ \\
$\gamma_{n m}^{j}$ & $h_{j m}^{\mathrm{P}}$ & 0 & $-\left(p_{j m}^{2}+q^{2}\right)$ \\
$k_{n m}^{j}$ & $h_{j m}^{\mathrm{P}}$ & $h_{j m}^{\mathrm{S}}$ & $h_{j m}^{\mathrm{S}}$ \\
\hline
\end{tabular}


Table 3. Parameters for the embankment and banks ( $j=\mathrm{e}, \mathrm{b}^{ \pm}$)

\begin{tabular}{cc}
\hline Lamé constant, $\lambda_{j}$ & $8.7 \times 10^{7} \mathrm{~N} / \mathrm{m}^{2}$ \\
Shear modulus, $\mu_{j}$ & $6.48 \times 10^{6} \mathrm{~N} / \mathrm{m}^{2}$ \\
Mass density, $\rho_{j}$ & $1800 \mathrm{~kg} / \mathrm{m}^{3}$ \\
Hysteretic damping ratio, $\delta_{j}$ & 0.13 \\
Half load width $a$ & $1 \mathrm{~m}$ \\
Half load length $b$ & $1 \mathrm{~m}$ \\
$a_{\mathrm{e}}$ & $7 \mathrm{~m}$ \\
$d_{\mathrm{e}}$ & $3 \mathrm{~m}$ \\
$b_{1}$ & $9 \mathrm{~m}$ \\
$b_{2}$ & $50 \mathrm{~m}$ \\
$L$ & $8 \mathrm{~m}$ \\
\hline
\end{tabular}


Table 4. Parameters for fully water-saturated poroelastic soil medium

Shear modulus, $\mu$

Poisson's ratio, $v$

Water density, $\rho_{\mathrm{f}}$

Solid density, $\rho_{\mathrm{s}}$

Porosity, $n$

Coefficient of material damping, $D$

Permeability coefficient, $k_{\mathrm{d}}$

The parameter for the compressibility of the soil particle, $\alpha$

The parameter for the compressibility of the fluid, $M$

$6.0 \times 10^{6} \mathrm{~N} / \mathrm{m}^{2}$
0.35
$1000 \mathrm{~kg} / \mathrm{m}^{3}$
$1816 \mathrm{~kg} / \mathrm{m}^{3}$
0.4
0.05
$10^{-5} \mathrm{~m} / \mathrm{s}$
0.97
$7.2 \times 10^{8} \mathrm{~N} / \mathrm{m}^{2}$

